Pacific Journal of Mathematics

ADJOINT QUASI-DIFFERENTIAL OPERATORS OF EULER 


\title{
ADJOINT QUASI-DIFFERENTIAL OPERATORS OF EULER TYPE
}

\author{
JOHN S. BRADLEY
}

This paper treats linear quasi-differential operators of the form

$$
L[y]=\sum_{j=0}^{n} p_{0 j} y^{(\jmath)}-\left(\sum_{j=0}^{n} p_{1 \jmath} y^{(\jmath)}-\left(\cdots-\left(\sum_{j=0}^{n} p_{m \jmath} y^{(j)}\right)^{\prime} \cdots\right)^{\prime}\right)^{\prime},
$$

based on an integrable $(m+1) \times(n+1)$ matrix function $\left[p_{i j}\right]$, $(i=0, \cdots, m ; j=0, \cdots, n)$, about which suitable regularity assumptions are made. Results obtained by Reid (Trans. Amer. Math. Soc. Vol. 85 (1957), pp. 446-461) are extended to operators of the type considered here.

A generalized Green's function for the system $\{L[y]=0$, $y \in \mathscr{D}\}$ is defined, where $\mathscr{D}$ is a linear subspace of the domain of $L$. Resolvent and deterministic properties of this function are presented, together with the relationship of such a generalized Green's function to the generalized Green's function for the associated adjoint system.

For a large class of two-point boundary problems in which the boundary conditions involve the characteristic parameter linearly it is shown that there exists a simultaneous canonical representation of the boundary conditions for a given problem and those of its adjoint; in particular, in the self-adjoint case this canonical representation has the form of boundary conditions and transversality conditions for a variational problem. Finally, these results are applied to a two-point boundary problem involving a differential operator of the type considered in the paper of Reid above.

Since an important example of an operator of the form of $L[y]$ is the Euler operator in the calculus of variations, we shall refer to such operators as quasi-differential operators of Euler type.

Section 2 gives a more precise description of the operator, and Section 3 is concerned with a discussion of its adjoint. In particular it is shown that if $\mathscr{D}_{0}$ is the class of functions $y$ in the domain of $L$ with the property that the functions $y, y^{\prime}, \cdots, y^{(n-1)}, \widetilde{y}_{m} \equiv \sum_{j=0}^{n} p_{m j} y^{(j)}$, $\widetilde{y}_{i} \equiv \sum_{j=0}^{n} p_{i j} y^{(j)}-\widetilde{y}_{i+1}^{\prime},(i=m-1, \cdots, 1)$, vanish at $a$ and at $b$, and if $T_{0}$ is the restriction of $L$ to $\mathscr{D}_{0}$, then the adjoint operator $T_{0}^{*}$ is given by

Received May 21, 1964. The author wishes to express his sincere appreciation to Professor W. T. Reid for his valuable suggestions and assistance throughout the preparation of this $\mathrm{Ph}$. D. dissertation (State University of Iowa, June 1964). The research for this paper was supported by the Air Force Office of Scientific Research, under grants AF-AFOR-62-78 and AF-AFOR-438-63. The authors address is now: Department of Mathematics, University of Tennessee, Knoxville. 


$$
T_{0}^{*}[z]=L^{\text {糸 }}[z] \equiv \sum_{i=0}^{m} \bar{p}_{i 0} z^{(i)}-\left(\sum_{i=0}^{m} \bar{p}_{i 1} z^{(\imath)}-\left(\cdots-\left(\sum_{i=0}^{m} \bar{p}_{i n} z^{(i)}\right)^{\prime} \cdots\right)^{\prime}\right)^{\prime} .
$$

Section 4 is a study of extensions of the operator $T_{0}$, and their adjoints. Section 5 is devoted to generalized Green's functions for Euler type quasi-differential systems and their adjoints, and extends the results of Elliott [3] and Reid [5] to the case where the number of linearly independent boundary conditions may differ from the order of the differential equation.

Section 6 is concerned with a certain class of two-point boundary problems in which the boundary conditions involve the characteristic parameter linearly. It is shown that there exists a simultaneous canonical representation of the boundary conditions for a given problm and those of its adjoint; in particular, in the self-adjoint case this canonical representation has the form of boundary conditions and transversality conditions for a variational problem.

Finally, $\S 7$ is devoted to an application of the results of $\S 6$ to a two-point boundary problem involving a differential operator of the type considered by Reid in [7].

The symbol $\mathfrak{F}_{n},(n=0,1,2, \cdots)$, will signify the class of complexvalued functions defined on the compact interval $[a, b]$ which have $n$ continuous derivatives. The set of functions $y$ in $\mathfrak{E}_{n-1}$ for which $y^{(n-1)}$ is a.c. (absolutely continuous) is denoted by $\mathfrak{U}_{n},(n=0,1,2, \cdots)$. In particular, $\mathfrak{S}_{0}$ and $\mathfrak{A}_{0}$ will signify respectively the classes of continuous and Lebesgue integrable complex-valued functions defined on $[a, b]$. If $f$ and $g$ belong to $\mathfrak{A}_{0}$ and $f(x)=g(x)$ almost everywhere, we will simply write $f=g$. If $f$ is a complex-valued function on $[a, b]$, then $\bar{f}$ denotes the function with domain $[a, b]$ whose value at $x$ is the complex conjugate of $f(x)$. If $u$ and $v$ are functions on $[a, b]$ and $\bar{v} u \in \mathfrak{A}_{0}$, then we define $(u, v)$ as

$$
(u, v)=\int_{a}^{b} \bar{v} u \text {. }
$$

Matrix notation will be used except where it is impracticable. If $M$ is a matrix, then the conjugate transpose of $M$ is denoted by $M^{*}$. Vectors are treated as matrices with one column. The symbols $E_{n}$ and $0_{m n}$ are used to represent the $n \times n$ identity matrix and the $m \times n$ zero matrix, respectively; the subscripts will be omitted when there is no danger of confusion.

A matrix function is said to be continuous, integrable, etc. whenever each of its elements possesses the specified property. If $A$ is an a.c. matrix function, then $A^{\prime}(x)$ signifies the matrix of derivatives at values for which these derivatives exist and the zero matrix elsewhere.

2. Description of the operator. Suppose that $\left[p_{i j}\right],(i=0, \cdots$, 
$m \geqq 1 ; j=0, \cdots, n \geqq 1)$, is an integrable $(m+1) \times(n+1)$ matrix function on a compact interval $[a, b]$ and that $p_{o n}$ and $p_{m o}$ are essentially bounded. For suitable $y$ in $\mathfrak{U}_{n}$ define functions $\widetilde{y}_{1}, \cdots, \widetilde{y}_{m}$ as follows:

$$
\begin{aligned}
& \widetilde{y}_{m}(x)=\sum_{j=0}^{n} p_{m j}(x) y^{(j)}(x) ; \\
& \text { if } \widetilde{y}_{j+1} \in \mathfrak{X}_{1}, \text { then } \widetilde{y}_{i}(x)=\sum_{j=0}^{n} p_{i j}(x) y^{(j)}(x)-\widetilde{y}_{i+1}^{\prime}(x), \\
& (i=m-1, \cdots, 1) .
\end{aligned}
$$

The class of functions $y$ in $\mathfrak{A}_{n}$ for which $\widetilde{y}_{1}, \cdots, \widetilde{y}_{m}$ are a.c. will be denoted by $\widetilde{\mathfrak{A}}_{n}$. For convenience the vector functions $\left(y^{(j-1)}\right)$, $(j=1, \cdots, n)$, and $\left(\widetilde{y}_{i}\right),(i=1, \cdots, m)$, will be denoted by $\hat{y}$ and $\widetilde{y}$, respectively; the $(n+m)$-vector function $\left(y, \cdots, y^{(n-1)}, \widetilde{y}_{1}, \cdots, \widetilde{y}_{m}\right)$ will be represented by $\bar{y}$.

Denote by $L$ the operator with domain $\widetilde{\mathfrak{A}}_{n}$ which is defined by

$$
L[y]=\sum_{j=0}^{n} p_{o j} y^{(j)}-\tilde{y}_{1}^{\prime} .
$$

The operator $L$ is a quasi-differential operator in the sense of Bôcher [1]; in particular, it is a generalization of the Euler operator in the calculus of variations and, as was stated in the introduction, it will be called a quasi-differential operator of the Euler type.

Let $\tilde{\mathfrak{A}}_{n}^{0}$ be the collection of functions $y$ in $\widetilde{\mathfrak{A}}_{n}$ for which $\bar{y}(\alpha)=0=$ $\widehat{y}(b)$, and denote by $T_{0}$ the restriction of $L$ to $\widetilde{\mathfrak{V}}_{n}^{0}$. Suppose that $\mathscr{D}_{0}^{*}$ is the class of functions $z$ in $\mathfrak{A}_{0}$ which are essentially bounded and have the property that there exists a function $f_{z}$ in $\mathfrak{A}_{0}$ such that $(L[y], z)=\left(y, f_{z}\right)$ for all $y$ in $\tilde{\mathfrak{A}}_{n}^{0}$.

A second operator $L^{\downarrow}$ will now be defined. For suitable functions $z$ in $\mathfrak{A}_{m}$ define functions $\widetilde{z}_{1}, \cdots, \widetilde{z}_{n}$ as follows:

$$
\begin{aligned}
& \widetilde{z}_{n}(x)=\sum_{i=0}^{m} \bar{p}_{i n}(x) z^{(i)}(x) ; \\
& \text { if } \widetilde{z}_{j+1} \in \mathfrak{A}_{1}, \text { then } \widetilde{z}_{j}(x)=\sum_{i=0}^{m} \bar{p}_{i j}(x) z^{(i)}(x)-\widetilde{z}_{j+1}^{\prime}(x), \\
& (j=n-1, \cdots, 1) .
\end{aligned}
$$

The class of functions $z$ in $\mathfrak{A}_{m}$ for which $\widetilde{z}_{1}, \cdots, \widetilde{z}_{n}$ are a.c. will be denoted by $\widetilde{\mathfrak{A}}_{m}$. Let $L$ be the operator with domain $\widetilde{\mathfrak{A}}_{m}$ defined by

$$
L^{\text {幽}}[z]=\sum_{i=0}^{m} \bar{p}_{i o} z^{(i)}-\widetilde{z}_{1}^{\prime} .
$$

If $z \in \widetilde{\mathfrak{A}}_{m}$, then $\check{z}$ and $\widetilde{z}$ will signify the vector functions $\left(z^{(i-1)}\right)$, $(i=1, \cdots, m)$, and $\left(\widetilde{z}_{j}\right),(j=1, \cdots, n)$, respectively. The $(m+n)$ vector function $\left(z, \cdots, z^{(m-1)}, \widetilde{z}_{1}, \cdots, \widetilde{z}_{n}\right)$ will be denoted by $\breve{z}$. 
Except when a statement is made to the contrary, the following hypothesis will be assumed throughout this paper.

Hypothesis (H). The matrix $\left[p_{i j}(x)\right],(i=0, \cdots, m ; j=0, \cdots, n)$, is integrable and there exists an $\varepsilon>0$ such that $\left|p_{m n}(x)\right| \geqq \varepsilon$ almost everywhere on $[a, b]$. Moreover, $p_{0 n}$ and $p_{m 0}$ are essentially bounded and $p_{i n} p_{m n}^{-1} p_{m j}$ is integrable, $(i=1, \cdots, m-1 ; j=1, \cdots, n-1)$.

It is to be noted that if $y \in \widetilde{\mathfrak{A}}_{n}$ and $z \in \widetilde{\mathfrak{A}}_{m}$, then $L[y]$ and $L^{\natural[}[z]$ are integrable.

Let $\mathscr{A}_{1}(x), \mathscr{A}_{2}(x), \mathscr{A}_{3}(x)$, and $\mathscr{A}_{4}(x)$ be $m \times n, m \times m, n \times n$, and $n \times m$ matrices, respectively, defined as follows:

$$
\begin{aligned}
& \mathscr{A}_{1}(x)= {\left[p_{i j}(x)-p_{i n}(x) p_{m n}^{-1}(x) p_{m j}(x)\right], } \\
& \mathscr{A}_{2}(x)= {\left[\begin{array}{cc}
0_{1 m-1} & p_{0 n}(x) p_{m n}^{-1}(x) \\
-E_{m-1} & p_{i n}(x) p_{m n}^{-1}(x)
\end{array}\right], \quad(i=1, \cdots, m-1), } \\
& \mathscr{A}_{3}(x)= {\left[\begin{array}{cc}
0_{n-11} & -E_{n-1} \\
p_{m n}^{-1}(x) p_{m 0}(x) & p_{m n}^{-1}(x) p_{m j}(x)
\end{array}\right], \quad(j=1, \cdots, n-1), } \\
& \mathscr{A}_{4}(x)=\left[\begin{array}{cc}
0_{n-1} & 0_{n-11} \\
0_{1 m-1} & -p_{m n}^{-1}(x)
\end{array}\right] .
\end{aligned}
$$

If $f$ and $g$ belong to $\mathfrak{A}_{0}$, then the equation $L[y]=f$ is equivalent to the following system in the vector functions $\hat{y}=\left(\hat{y}_{i}\right),(i=1, \cdots, n)$, and $\widetilde{y}=\left(\widetilde{y}_{j}\right),(j=1, \cdots, m)$ :

$$
\begin{aligned}
& \hat{y}^{\prime}+\mathscr{A}_{3} \hat{y}+\mathscr{A}_{4} \widetilde{y}=0, \\
& \widetilde{y}^{\prime}-\mathscr{A}_{1} \hat{y}-\mathscr{A}_{2} \widetilde{y}=-f e^{(m, 1)} ;
\end{aligned}
$$

and the equation $L^{\text {म }}[z]=g$ is equivalent to the following system in the vector functions $\check{z}=\left(\check{z}_{j}\right),(j=1, \cdots, m)$, and $\widetilde{z}=\left(\widetilde{z}_{j}\right),(i=1, \cdots, n)$ :

$$
\begin{aligned}
& \check{z}^{\prime}+\mathscr{A}_{2} * \check{z}+\mathscr{A}_{4} * \widetilde{z}=0, \\
& \widetilde{z}^{\prime}-\mathscr{A}_{1} * \check{z}-\mathscr{A}_{3} * \widetilde{z}=-g e^{(n, 1)},
\end{aligned}
$$

where $e^{(k, 1)},(k=1,2,3, \cdots)$, is used to denote the $k$-dimensional vector whose first coordinate is one, and whose remaining coordinates are zero. If $\mathscr{J}$ is the $(m+n) \times(m+n)$ matrix

$$
\mathscr{J}=\left[\begin{array}{cc}
0_{m n} & -E_{m} \\
E_{n} & 0_{n m}
\end{array}\right],
$$

and $\mathscr{A}$ is the $(m+n) \times(m+n)$ matrix function defined by 


$$
\mathscr{A}(x)=\left[\begin{array}{ll}
\mathscr{A}_{1}(x) & \mathscr{A}_{2}(x) \\
\mathscr{A}_{3}(x) & \mathscr{A}_{4}(x)
\end{array}\right]
$$

then (2.5) and (2.6) may be written as

$$
\mathscr{L}[\hat{y}] \equiv \mathscr{J} \bar{y}^{\prime}+\mathscr{A} \hat{y}=f e^{(m+n, 1)},
$$

and

$$
\mathscr{S}^{4}[\bar{z}] \equiv-\mathscr{J}^{*} \bar{z}+\mathscr{A}^{*} \bar{z}=g e^{(m+n, 1)} \text {, }
$$

respectively.

Theorems on existence and uniqueness of solutions of $L[y]=f$ and $L^{\text {幺 }}[z]=g$ follow from corresponding theorems for the respective first order systems (2.8) and (2.9). It also follows that $y \in \widetilde{\mathfrak{A}}_{n}$ if and only if there exists an integrable function $f$ such that $y$ is the first coordinate of a vector function $\widehat{y}$ satisfying (2.8), and $z \in \widetilde{\mathfrak{A}}_{m}$ if and only if there is an integrable function $g$ such that $z$ is the first coordinate of a vector function $\bar{z}$ satisfying (2.9).

The differential system (2.5) is identically normal in the sense that if $\bar{y}(x)$ is a solution of $\mathscr{L}[\hat{y}]=0$ with $\widehat{y}(x) \equiv 0$ on a subinterval $X$ of $[a, b]$, then $\widehat{y}(x) \equiv 0$ on $X$. Indeed, if $\hat{y}$ is such a solution of (2.5), then $\widetilde{y}$ is a solution of $\widetilde{y}^{\prime}-\mathscr{A}_{2} \widetilde{y}=0$ satisfying $\mathscr{A}_{4} \widetilde{y}=0$ on $X$. This latter condition implies that $\widetilde{y}_{m}(x) \equiv 0$ on this subinterval, and the differential equation $\widetilde{y}^{\prime}-\mathscr{A}_{2} \widetilde{y}=0$ implies in turn that $\widetilde{y}_{j}(x) \equiv 0$ on $X$ for $j=m-1, \cdots, 1$. Similarly, system (2.6) is also identically normal. It follows from the identical normality of (2.5) that functions $y_{\alpha}$ in $\widetilde{\mathfrak{A}}_{n}$ are linearly independent solutions of $L[y]=0$ if and only if the corresponding vector functions $\bar{y}_{\alpha}$ are linearly independent solutions of $\mathscr{L}[\hat{y}]=0$. Similarly, it follows from the identical normality of (2.6) that functions $z_{\alpha}$ in $\tilde{\mathfrak{A}}_{m}$ are linearly independent solutions of $L^{4}[z]=0$ if and only if the corresponding vector functions $\breve{z}_{\alpha}$ are linearly independent solutions of $\mathscr{S}^{\text {tr }}[\bar{z}]=0$.

3. The adjoint operator. If $\mathscr{J}$ is the $(m+n) \times(m+n)$ matrix defined as in (2.7), then we may establish the following Lagrange identity by a simple inductive argument which does not use hypothesis $(\mathrm{H})$.

LEMmA 3.1. If $y \in \widetilde{\mathfrak{A}}_{n}$ and $z \in \widetilde{\mathfrak{A}}_{m}$, then

$$
\bar{z} L[y]-\bar{L}^{\natural}[z] y=\left(\bar{z}^{*} \mathscr{J} \bar{y}\right)^{\prime} .
$$

THEOREM 3.1. If $f \in \mathfrak{A}_{0}$, then there exists a $y$ in $\widetilde{\mathfrak{A}}_{n}^{0}$ such that $L[y]=f$ if and only if $z$ in $\widetilde{\mathfrak{A}}_{m}$ and $L^{\text {म }}[z]=0$ implies that $(f, z)=0$. 
Now if $y \in \widetilde{\mathfrak{A}}_{n}^{0}, L[y]=f, z \in \widetilde{\mathfrak{A}}_{m}$, and $L^{\text {讦 }}[z]=0$, then, in view of Lemma 3.1,

$$
(f, z)=(L[y], z)-\left(y, L^{\natural}[z]\right)=\left.\breve{z}^{*} \mathcal{J} \hat{y}\right|_{a} ^{b}=0 .
$$

On the other hand, suppose that $(f, z)=0$ whenever $z \in \widetilde{\mathfrak{A}}_{m}$ and $L^{\text {मn }}[z]=0$, and let $y$ be the function in $\tilde{\mathfrak{A}}_{n}$ such that $L[y]=f$ and $\bar{y}(a)=0$. If $z_{j},(j=1, \cdots, m+n)$ are linearly independent solutions of $L[z]=0$, then the $(m+n) \times(m+n)$ matrix $\bar{Z}(x)$ with column vectors $\breve{z}_{j}(x), \quad(j=1, \cdots, m+n)$, is nonsingular on $[a, b]$. From Lemma 3.1 we have the vector equation

$$
0=\left[\left(f, z_{j}\right)-\left(y, L^{\sharp}\left[z_{j}\right]\right)\right]=\left.\breve{Z}^{*} \mathscr{J} \widehat{y}\right|_{a} ^{b}=\breve{Z}^{*}(b) \mathscr{J} \bar{y}(b),
$$

and consequently $\bar{y}(b)=0$ also.

THEOREM 3.2. If hypothesis $(\mathrm{H})$ holds, then $\mathscr{D}_{0}^{*}=\breve{\mathfrak{A}}_{m}$ and $f_{z}=$ $L^{\sharp}[z]$ on $\mathscr{D}_{0}^{*}$.

That $\widetilde{\mathfrak{A}}_{m} \subset \mathscr{D}_{0}^{*}$ follows from Lemma 3.1. Now let $z_{0} \in \mathscr{D}_{0}^{*}$ and suppose $f_{z_{0}}$ is a corresponding function in $\mathfrak{A}_{0}$ such that $\left(L[y], z_{0}\right)=$ $\left(y, f_{z_{0}}\right)$ when $y \in \widetilde{\mathfrak{A}}_{n}^{0}$. Choose $w_{0}$ in $\widetilde{\mathfrak{A}}_{m}$ such that $L$ 出 $\left[w_{0}\right]=f_{z_{0}}$, and suppose that $z_{i} \in \widetilde{\mathfrak{A}}_{m}$ are linearly independent solutions of $L^{\text {in }}\left[z_{i}\right]=0$, with $\left(z_{i}, z_{j}\right)=\delta_{i j},(i, j=1, \cdots, m+n)$. If $w=w_{0}+\sum_{j=1}^{m+n}\left(z_{0}-w_{0}, z_{j}\right) z_{j}$, then $L^{\sharp}[w]=f_{z 0}$ and $\left(z_{0}-w, z\right)=0$ when $z \in \widetilde{\mathfrak{A}}_{m}$ and $L^{\sharp}[z]=0$. It follows that if $y \in \widetilde{\mathfrak{A}}_{n}^{0}$, then

$$
\left(L[y], z_{0}\right)=\left(y, f_{z_{0}}\right)=\left(y, L^{\natural}[w]\right)=(L[y], w) ，
$$

so that $\left(L[y], z_{0}-w\right)=0$ when $y \in \tilde{\mathfrak{A}}_{n}^{0}$. But it follows from Theorem 3.1 that there is a function $y$ in $\tilde{\mathfrak{A}}_{a}^{0}$ such that $L[y]=z_{0}-w$. Consequently $\left(z_{0}-w, z_{0}-w\right)=0$ and $z_{0}=w \in \widetilde{\mathfrak{A}}_{m}$, so that $\mathscr{D}_{0}^{*}=\widetilde{\mathfrak{A}}_{m}$ and $f_{z_{0}}=L^{\Downarrow}\left[z_{0}\right]$. This result extends Theorem 4.1 of Reid [7].

Now the operator $T_{0}^{*}$ adjoint to $T_{0}$ is defined to be the operator on $\mathscr{D}_{0}^{*}$ with value $f_{z}$ at $z$. In view of Theorem 3.2 we have $\mathscr{D}_{0}^{*}=$ $\widetilde{\mathfrak{A}}_{m}$ and $T_{0}^{*}[z]=L^{\star}[z]$.

4. Extensions of the operator $T_{0}$. Let $\mathscr{D}$ be a linear subspace of $\widetilde{\mathfrak{A}}_{n}$ containing $\widetilde{\mathfrak{A}}_{n}^{0}$, and denote by $T$ the restriction of $L$ to $\mathscr{D}$. Denote by $\mathscr{D}^{*}$ the class of functions $z$ in $\mathfrak{A}_{0}$ which are essentially bounded and for which there exists an $f_{z}$ in $\mathfrak{A}_{0}$ such that $(L[y], z)=$ $\left(y, f_{z}\right)$ for all $y$ in $\mathscr{D}$. It follows from Theorem 3.2 that $\mathscr{D}^{*} \subset \widetilde{\mathfrak{A}}_{m}$ and for each $z$ in $\mathscr{D}^{*}$ there is at most one $f_{z}$, namely $L^{\text {in }}[z]$, such that $(L[y], z)=\left(y, f_{z}\right)$ for all $y$ in $\mathscr{D}$. The adjoint $T^{*}$ of $T$ is the 
operator on $\mathscr{D}^{*}$ defined by the formula $T^{*}[z]=f_{z}$. The operator $T$ is said to be self-adjoint if and only if $\mathscr{D}=\mathscr{D}^{*}$ and $T=T^{*}$.

The following lemma will be helpful in describing $\mathscr{D}^{*}$. If $y_{j} \in \widetilde{\mathfrak{A}}_{n}$, $(j=1, \cdots, m+n)$, then $\hat{Y}$ will denote the matrix function defined by $\bar{Y}(x) \equiv\left[\bar{y}_{j}(x)\right],(j=1, \cdots, m+n)$.

LEMMA 4.1. If $\eta$ and $\zeta$ are $(m+n)$-vectors, then there exists a function $y \in \widetilde{\mathfrak{A}}_{n},\left(z \in \widetilde{\mathfrak{A}}_{m}\right)$, such that $\widehat{y}(a)=\eta$ and $\bar{y}(b)=\zeta,(\widehat{z}(a)=\eta$ $d a n \bar{z}(b)=\zeta)$.

Since $\widetilde{\mathfrak{A}}_{n}$ is a vector space it is enough to show that there exist $m+n$ functions $y_{j}$ in $\widetilde{\mathfrak{A}}_{n}$ such that $\widehat{y}_{j}(a)=0, \quad(j=1, \cdots, m+n)$ while $\hat{Y}(b)$ is nonsingular, and to show a corresponding result with $a$ and $b$ interchanged. To establish the existence of functions $y_{j}$ in $\tilde{\mathfrak{A}}_{n}$ such that $\widehat{y}_{j}(a)=0,(j=1, \cdots, m+n)$, and $\bar{Y}(b)$ is nonsingular, suppose to the contrary that for each collection of $m+n$ functions $y_{j}$ in $\widetilde{\mathfrak{A}}_{n}$ satisfying $\widehat{y}_{j}(a)=0,(j=1, \cdots, m+n)$, we have $\bar{Y}(b)$ singular. Let $z_{j}$ be $m+n$ linearly independent solutions of $L^{4}[z]=0$, and for $j=1, \cdots, m+n$ let $y_{j}$ be the function in $\widetilde{\mathfrak{A}}_{n}$ such that $L\left[y_{j}\right]=z_{j}$ and $\bar{y}_{j}(a)=0$. Then there is a nonzero $(m+n)$-vector $\xi=\left(\xi_{j}\right)$ such that $\bar{Y}(b) \xi=0$. If $y(x)=\sum_{j=1}^{m+n} y_{j}(x) \xi_{j}$ and $z(x)=\sum_{j=1}^{m+n} z_{j}(x) \xi_{j}$, then $L[y]=z, L^{\natural}[z]=0$ and $z(x) \not \equiv 0$, moreover, $y \in \widetilde{\mathfrak{A}}_{n}^{0}$. Hence it follows from Lemma 3.1 that

$$
0=(L[y], z)-\left(y, L^{\sharp}[z]\right)=(z, z),
$$

which is impossible since $z(x) \not \equiv 0$. The numbers $a$ and $b$ may be interchanged and the preceding argument remains valid. The result for $\widetilde{\mathfrak{A}}_{m}$ follows by interchanging the roles of $\widetilde{\mathfrak{A}}_{n}$ and $\widetilde{\mathfrak{A}}_{m}$, that is, by replacing $\left[p_{i j}\right]$ with $\left[p_{i j}\right]^{*}$.

Denote by $\mathscr{B}$ the subspace of $2(m+n)$-dimensional complex space consisting of the end values $(\widehat{y}(a), \widetilde{y}(a), \widehat{y}(b), \widetilde{y}(b))$ for functions $y$ in $\mathscr{D}$. Similarly, $\mathscr{B}^{*}$ will denote the subspace of end values $(\check{z}(a), \widetilde{z}(a), \check{z}(b), \widetilde{z}(b))$ for functions $z$ in $\mathscr{D}^{*}$. If $k<2 m+2 n$ and the dimension of $\mathscr{B}$ is $2 m+2 n-k$, then let $P$ and $Q$ be $(m+n) \times(2 m+2 n-k)$ matrices such that the columns of $\left[-P^{*} Q^{*}\right]^{*}$ form a basis for $\mathscr{B}$. If $k>0$ also, then let $M$ and $N$ be $k \times(m+n)$ matrices such that the $k \times 2(m+n)$ matrix $[M N]$ has rank $k$ and $M P-N Q=0$. Then in view of Lemma 4.1 we have that $\mathscr{D}$ is characterized as the class of functions $y$ in $\widetilde{\mathfrak{A}}_{n}$ with the property that

$$
s(\widehat{y}) \equiv M \widehat{y}(a)+N \widehat{y}(b)=0 .
$$

If $k=0$, then by Lemma 4.1 we have $\mathscr{D}=\widetilde{\mathfrak{A}}_{n}$. 
THEOREM 4.1. Dim $\mathscr{B}+\operatorname{dim} \mathscr{B}^{*}=2 m+2 n$; if $\operatorname{dim} \mathscr{B}>0$ and $P, Q$ are $(m+n) \times(2 m+2 n-k)$ matrices such that the column vectors of $\left[-P^{*} Q^{*}\right]^{*}$ from a basis for $\mathscr{B}$, then $\mathscr{D}^{*}$ is the class of functions $z$ in $\tilde{\mathfrak{A}}_{m}$ for which

$$
P^{*} \mathscr{J} * \breve{z}(a)+Q^{*} \mathscr{J} * \breve{z}(b)=0 \text {. }
$$

First note that if $\operatorname{dim} \mathscr{B}=0$, then $\mathscr{D}^{*}=\widetilde{\mathfrak{A}}_{m}$ by Theorem 3.2 , and thus by Lemma 4.1 we have $\operatorname{dim} \mathscr{B}^{*}=2 m+2 n$. Now suppose that $\operatorname{dim} \mathscr{B}>0, z \in \widetilde{\mathscr{A}}_{m}$, and (4.2) holds. Then for $y$ in $\mathscr{D}$ and $\xi$ a $(2 m+2 n-k)$-vector chosen so that $\bar{y}(a)=-P \xi$ and $\bar{y}(b)=Q \xi$ it follows from Lemma 3.1 that

$$
(L[y], z)-\left(y, L^{\sharp}[z]\right)=\left.\breve{z}^{*} \mathscr{J} \bar{y}\right|_{a} ^{b}=\left\{P^{*} \mathscr{J}^{*} \breve{z}(a)+Q^{*} \mathscr{J} * \bar{z}(b)\right\}^{*} \xi=0
$$

and hence $z \in \mathscr{D}^{*}$. On the other hand, if $z \in \mathscr{D}^{*}$ then it follows from Theorem 3.2 that $z \in \widetilde{\mathfrak{A}}_{m}$, since $\widetilde{\mathfrak{A}}_{n}^{0} \subset \mathscr{D}$. Then (4.2) follows from Lemma 3.1, Lemma 4.1 and the choice of $P$ and $Q$. Therefore, in view of Lemma 4.1 , it follows that $\operatorname{dim} \mathscr{B}+\operatorname{dim} \mathscr{B}^{*}=2 m+2 n$.

CoRollary I. If $\operatorname{dim} \mathscr{B}>0$, and $R$ and $S$ are $(2 m+2 n-k) \times$ $(m+n)$ matrices, then $\mathscr{D}^{*}$ is the collection of functions $z$ in $\widetilde{\mathfrak{A}}_{m}$ for which

$$
R \breve{z}(a)+S \breve{z}(b)=0
$$

if and only if the $(2 m+2 n-k) \times 2(m+n)$ matrix $[R S]$ has rank $2 m+2 n-k$ and $M \mathscr{J}^{*} R^{*}-N \cdot \mathscr{J}^{*} S^{*}=0$.

COROLLARY II. The adjoint of $T^{*}$ is $T$.

The index of compatibility for a system $L[y]=0, y \in \mathscr{D}$ is defined to be $\operatorname{dim}\{y: y \in \mathscr{D}$ and $L[y]=0\}$. The next two theorems are consequences of the equivalence of the equations $L[y]=f$ and $L$ t $[z]=g$ to the systems (2.8) and (2.9), respectively, and corresponding theorems on first order systems. Analogous theorems for $n$th order linear differential equations are given in [2, Chapter 11], and those results may be extended to first order systems.

THEOREM 4.2. If $\operatorname{dim} \mathscr{B}^{*}=k$ and the index of compatibility of the system $L[y]=0, y \in \mathscr{D}$ is $r$, then $\rho=k+r-m-n$ is the index of compatibility for the system $L^{\text {म }}[z]=0, z \in \mathscr{D}^{*}$.

THEOREM 4.3. If $f \in \mathfrak{A}_{0}$, then there exists a function $y$ in $\mathscr{D}$ such that $L[y]=f$ if and only if $(f, z)=0$ for all $z$ in $\mathscr{D}^{*}$ satisfying $L^{\text {出}}[z]=0$. 
The next two theorems are analogues of Theorems 6.1 and 6.2 of Reid [7]. The second of the two gives necessary and sufficient conditions for the operator $T$ to be self-adjoint when $\left[p_{i j}(x)\right]$ is Hermitian. If $y_{j} \in \widetilde{\mathfrak{A}}_{n}$ and $\bar{Y}=\left[\widehat{y}_{j}\right], \quad(j=1, \cdots, m+n)$, then the symbols $s(\bar{Y})$ and $s^{-}(\bar{Y})$ are used for the $k \times(m+n)$ matrices $M \hat{Y}(a)+N \hat{Y}(b)$ and $M \widehat{Y}(a)-N \widehat{Y}(b)$, respectively. Similarly, if $z_{j} \in \widetilde{\mathfrak{A}}_{m}$ and $\breve{Z}=\left[\breve{z}_{j}\right]$, $(j=1, \cdots, m+n)$, then $t(\breve{Z})$ and $t^{-}(\breve{Z})$ denote $R \breve{Z}(a)+S \grave{Z}(b)$ and $R \breve{Z}(a)-S \breve{Z}(b)$, respectively.

THEOREM 4.4. Suppose that $2(m+n)>\operatorname{dim} \mathscr{B}>0, y_{j}$ and $z_{j}$, $(j=1, \cdots, m+n)$, are linearly independent solutions of $L[y]=0$ and $L^{\natural}[z]=0$, respectively, and let $\Delta=\left(\breve{Z}^{*} \mathscr{J} \bar{Y}\right)^{-1}$. Then $\Delta$ is constant on $[a, b]$ and $\mathscr{D}^{*}$ is the collection of functions $z$ in $\widetilde{\mathfrak{A}}_{m}$ satisfying (4.3) if and only if the $(2 m+2 n-k) \times 2(m+n)$ matrix [RS] has rank $2 m+2 n-k$ and

$$
s(\hat{Y}) \Delta\left\{t^{-}(\check{Z})\right\}^{*}+s^{-}(\bar{Y}) \Delta\{t(\breve{Z})\}^{*}=0 .
$$

TheOREM 4.5. Suppose that $m=n, \quad\left[p_{i j}(x)\right], \quad(i, j=0, \cdots, n$; $x \in[a, b])$, is Hermitian and $\operatorname{dim} \mathscr{B}=2 n$. Let $y_{j},(j=1, \cdots, 2 n)$, be linearly independent solutions of $L[y]=0$, and let $\Delta=\left(\hat{Y}^{*} \mathscr{J} \bar{Y}\right)^{-1}$. Then $\Delta$ is constant on $[a, b]$, and $T$ is self-adjoint if and only if the $2 n \times 2 n$ matrix $s^{-}(\bar{Y}) \Delta\{s(\bar{Y})\}^{*}$ is Hermitian.

5. Generalized Green's functions. The subspaces $\mathscr{D}, \mathscr{D}^{*}$ of $\widetilde{\mathfrak{A}}_{n}$ and $\tilde{\mathfrak{A}}_{m}$, respectively, and the subspaces $\mathscr{B}, \mathscr{B}^{*}$ of $2(m+n)$ dimensional complex space are as defined in $\S 4$. If $0<\operatorname{dim} \mathscr{B}<2 m+$ $2 n$, then the matrices $M, N, P$, and $Q$ are as specified in $\S 4$.

If $f \in \mathfrak{A}_{0}$ then we are concerned with solutions of the quasidifferential system

$$
L[y]=f, \quad y \in \mathscr{D} .
$$

Of prime importance is the homogeneous system

$$
L[y]=0, \quad y \in \mathscr{D},
$$

and its adjoint system

$$
L^{\text {幻 }}[z]=0, \quad z \in \mathscr{D}^{*} .
$$

By definition a generalized Green's function for the system (5.2) is an essentially bounded and measurable function $g$ on $\square \equiv$ $\{(x, t): a \leqq x \leqq b, a \leqq t \leqq b\}$ with the property that if $f$ is a function in $\mathfrak{A}_{0}$ for which (5.1) has a solution, then a particular solution $y$ 
of (5.1) is given by

$$
y(x)=\int_{a}^{b} g(x, t) f(t) d t
$$

Reid [5] has shown the existence of a generalized Green's matrix for a compatible first order system with two-point boundary conditions, where the number of independent boundary conditions is equal to the number of differential equations. If $\operatorname{dim} \mathscr{B}=m+n$, then Reid's results could be used to obtain a generalized Green's function for (5.2). In this section the existence and some properties of a generalized Green's function will be shown when $\operatorname{dim} \mathscr{B}$ is not necessarily equal to $m+n$. The technique used here may be modified to extend Reid's results to the case where the number of independent boundary conditions is different from the number of differential equations.

For a $\nu$ th order linear differential operator $\sum_{j=0}^{\nu} q_{j}(x) y^{(j)}$ with $q_{j} \in C_{j},(j=0,1, \cdots, \nu)$, and $q_{\nu}(x) \neq 0$, the generalized Green's function has been treated by Greub and Rheinboldt [4] and Wyler [10]; a more comprehensive treatment of an algebraic theory of operator solutions of boundary problems, which includes this case as a special instance, is given in Wyler [11].

LEMMA 5.1. If $y_{j},(j=1, \cdots, m+n)$, are linearly independent solutions of $L[y]=0$, then there exist $m+n$ linearly independent solutions $z_{j}$ of $L^{i}[z]=0$ such that

$$
\breve{Z}^{*} \mathscr{J} \hat{Y}=E_{m+n}
$$

This result follows from Lemma 3.1 and the existence and uniqueness theorems for the equations $\mathscr{L}[\hat{y}]=0$ and $\mathscr{L}^{4}[\bar{z}]=0$.

If $y_{j} \in \widetilde{\mathfrak{A}}_{n}$ and $z_{j} \in \tilde{\mathfrak{U}}_{m}, \quad(j=1, \cdots, m+n)$, then define matrix functions $\hat{Y}, \tilde{Y}, \check{Z}$, and $\widetilde{Z}$ as follows: $\hat{Y}(x)=\left[\widehat{y}_{j}(x)\right], \tilde{Y}(x)=\left[\widetilde{y}_{j}(x)\right]$, $\check{Z}(x)=\left[\check{z}_{j}(x)\right]$, and $\widetilde{Z}(x)=\left[\widetilde{z}_{j}(x)\right],(j=1, \cdots, m+n)$.

COROLlaRY. If $y_{j}$ and $z_{j},(j=1, \cdots, m+n)$, are as in Lemma 5.1, then

$$
\begin{aligned}
& \hat{Y}(x) \check{Z} *(x) \equiv 0_{n m}, \quad \hat{Y}(x) \widetilde{Z} *(x) \equiv E_{n}, \\
& \widetilde{Y}(x) \check{Z}^{*}(x) \equiv-E_{m}, \quad \tilde{Y}(x) \widetilde{Z}^{*}(x) \equiv 0_{m n} \text {. }
\end{aligned}
$$

THEOREM 5.1. If $\tau \in[a, b], \xi_{j}$ is a constant, $y_{\jmath}$ and $z_{j},(j=1, \cdots$, $m+n)$, are as in Lemma 5.1, then the solution $y$ of $L[y]=f$ satisfying $\widehat{y}(\tau)=\sum_{j=1}^{m+n} \widehat{y}_{j}(\tau) \xi_{j}$ is given by the first component of the vector 


$$
\widehat{y}(x)=\sum_{j=1}^{m+n} \bar{y}_{j}(x) \xi_{j}+\int_{\tau}^{x} \sum_{j=1}^{m+n} \widehat{y}_{j}(x) \bar{z}_{j}(t) f(t) d t .
$$

Indeed, if $\xi=\left(\xi_{j}\right),(j=1, \cdots, m+n)$, and we set $\hat{y}(x)=\hat{Y}(x) u(x)$, for $u$ an $(m+n)$-vector function, then $\bar{y}$ is a solution of $\mathscr{L}[\bar{y}]=f e^{(m \div n, 1)}$, $\hat{y}(\tau)=\hat{Y}(\tau) \xi$ if and only if

$$
\mathscr{J} \bar{Y}(x) u^{\prime}(x)=e^{(m+n, 1)} f(x), \quad u(\tau)=\xi .
$$

Hence $u^{\prime}(x)=\breve{Z}^{*}(x) e^{(m+n, 1)} f(x)$ and

$$
u(x)=\xi+\int_{\tau}^{x} \breve{Z} *(s) e^{(m+n, 1)} f(s) d s,
$$

from which the theorem follows.

Now suppose that $y_{j},(j=1, \cdots, m+n)$, are linearly independent solutions of $L[y]=0$ and that $z_{j},(j=1, \cdots, m+n)$, are chosen as in Lemma 5.1. If $\operatorname{dim} \mathscr{B}=2 m+2 n-k, k>0$, then $s(\hat{Y})$ and $s^{-}(\hat{Y})$ are $k \times(m+n)$ matrices defined as $s(\bar{Y})=M \bar{Y}(a)+N \bar{Y}(b)$ and $s^{-}(\hat{Y})=M \bar{Y}(a)-N \bar{Y}(b)$. If $r$ is the index of compatibility for (5.2), then $s(\bar{Y})$ has rank $m+n-r$. If $r>0$, then let $S$ be an $(m+n) \times r$ matrix with the property that $S^{*} S=E_{r}$ and $s(\bar{Y}) S=0$. If $r>m+n-k$, then $T$ will represent a $k \times(k-m-n+r)$ matrix such that $T^{*} T=$ $E_{k-m-n+r}$ and $T^{*} s(\bar{Y})=0$. It follows that the $(k+r) \times(k+r)$ matrix

$$
\left[\begin{array}{cc}
s(\hat{Y}) & T \\
S^{*} & 0
\end{array}\right]
$$

is nonsingular, and its inverse is of the form

$$
\left[\begin{array}{ll}
D & S \\
T^{*} & 0
\end{array}\right]
$$

The $(m+n) \times k$ matrix $D$ is the generalized reciprocal of $s(\bar{Y})$ in the sense of E. H. Moore, (see [9, Section 14]). If $r=0$, then the matrix $S$ does not appear, if $r=m+n-k$, then $T$ does not appear.

Now if $\operatorname{dim} \mathscr{B}<2(m+n)$, let $G(x, t)$ be the $(m+n) \times(m+n)$ matrix defined by

$$
\begin{aligned}
& G(x, t)=\frac{1}{2} \bar{Y}(x)\left[\frac{|x-t|}{x-t} E_{m+n}+D s^{-}(\bar{Y})\right] \breve{Z}^{*}(t), \quad x \neq t ; \\
& G(x, x)=\frac{1}{2} \bar{Y}(x) D s^{-}(\bar{Y}) \breve{Z}^{*}(x), \quad x \in[a, b] .
\end{aligned}
$$

If $\operatorname{dim} \mathscr{B}=2(m+n)$, let $G(x, t)$ be defined by 


$$
\begin{aligned}
& G(x, t)=\frac{1}{2} \frac{|x-t|}{x-t} \hat{Y}(x) \breve{Z}^{*}(t), \quad x \neq t ; \\
& G(x, x)=0, \quad x \in[a, b] .
\end{aligned}
$$

Let $g_{0}$ be the function with domain $\square$ whose value at $(x, t)$ is the element in the first row and first column of $G(x, t)$, that is

$$
\begin{aligned}
& g_{0}(x, t)=g_{0,1}(x, t)+g_{0,2}(x, t) \quad \text { if } \operatorname{dim} \mathscr{B}<2(m+n) \text {, } \\
& g_{0}(x, t)=g_{0,1}(x, t) \quad \text { if } \operatorname{dim} \mathscr{B}=2(m+n) \text {, }
\end{aligned}
$$

where

$$
\begin{aligned}
& g_{0,1}(x, t)=\frac{1}{2} \operatorname{sgn}(x-t) \sum_{i=1}^{m+n} y_{i}(x) \bar{z}_{i}(t), \\
& g_{0,2}(x, t)=\frac{1}{2} \sum_{i, j=0}^{m+n} y_{i}(x) \mathscr{K}_{i j} \bar{z}_{j}(t),
\end{aligned}
$$

provided $\left[\mathscr{K}_{i j}\right]$ is the matrix $D s^{-}(\bar{Y})$ and $\operatorname{sgn} u=|u| / u$ for $u \neq 0$, $\operatorname{sgn} 0=0$.

THEOREM 5.2. The function $g_{0}$ defined above is a generalized Green's function for (5.2).

If $\operatorname{dim} \mathscr{B}=2(m+n)$, then this result follows directly from Theorem 5.1. Now suppose that $\operatorname{dim} \mathscr{B}<2(m+n)$, and $f$ is an integrable function for which (5.1) has a solution. If $y$ is a solution of $L[y]=f$, then for a suitable vector $\xi$ one has

$$
\bar{y}(x)=\frac{1}{2}\left[\bar{Y}(x) \xi+\int_{a}^{x} \hat{Y}(x) \breve{Z}^{*}(t) e^{(m+n, 1)} f(t) d t-\int_{x}^{b} \bar{Y}(x) \breve{Z}^{*}(t) e^{(m+n, 1)} f(t) d t\right] .
$$

Thus, since (5.9) is the inverse of (5.8), it follows that $y$ is a solution of (5.1) if and only if

$$
T^{*} s^{-}(\bar{Y}) \int_{a}^{b} \breve{Z}^{*}(t) e^{(m+n, 1)} f(t) d t=0,
$$

and for some $r$-vector $\eta$ we have

$$
\xi=D s^{-}(\bar{Y}) \int_{a}^{b} \breve{Z}^{*}(t) e^{(m+n, 1)} f(t) d t+S \eta .
$$

Therefore,

$$
\begin{aligned}
\widehat{y}(x)=\frac{1}{2}\left[\bar{Y}(x) S \eta+\bar{Y}(x) D s^{-}(\bar{Y})\right. & \int_{a}^{b} \breve{Z} *(t) e^{(m+n, 1)} f(t) d t \\
& \left.+\int_{a}^{b} \bar{Y}(x) \frac{|x-t|}{x-t} \breve{Z} *(t) e^{(m+n, 1)} f(t) d t\right],
\end{aligned}
$$


from which the theorem follows since $\eta$ may be chosen to be zero.

The symbol $g_{0}^{(i, j)}$ will be used to signify the partial derivative $\partial^{i+j} g_{0} / \partial t^{j} \partial x^{i}$. Generalized partial derivatives $g_{0}^{\langle\alpha, \beta\rangle}$ will now be defined for $g_{0}$. If $\alpha<n$ and $\beta<m$, then $g_{0}^{\langle\alpha, \beta\rangle}(x, t)=g_{0}^{(\alpha, \beta)}(x, t)$. If $\alpha<n$, then $g_{0}^{\langle\alpha, m+j\rangle},(j=0, \cdots, n-1)$, is defined as follows:

$$
g_{0}^{\langle\alpha, m\rangle}(x, t)=\sum_{i=0}^{m} \bar{p}_{i n}(t) g_{0}^{(\alpha, i)}(x, t) ;
$$

if $g^{\langle\alpha, m-1+j\rangle}$ is a.c. in its second argument, then

$$
\begin{array}{r}
g_{0}^{\langle\alpha, m+j\rangle}(x, t)=\sum_{i=0}^{m} \bar{p}_{i n-j}(t) g_{0}^{\langle\alpha, i\rangle}(x, t)-\partial / \partial t g_{0}^{\langle\alpha, m-1+j\rangle}(x, t), \\
(j=1, \cdots, n-1) .
\end{array}
$$

If $\beta<m$, then $g_{0}^{\langle n+i, \beta\rangle},(i=0, \cdots, m-1)$, is defined as follows:

$$
g_{0}^{\langle n, \beta\rangle}(x, t)=\sum_{j=0}^{n} p_{m j}(x) g_{0}^{(j, \beta)}(x, t) ;
$$

if $\boldsymbol{g}^{\langle n-1+i, \beta\rangle}$ is a.c. in its first argument, then

$$
\begin{aligned}
g_{0}^{\langle n+i, \beta\rangle}(x, t)=\sum_{j=1}^{n} p_{m-i j}(x) g_{0}^{(j, \beta)}(x, t)-\partial / \partial x g_{0}^{\langle n-1+i, \beta\rangle}(x, t) \\
(i=1, \cdots, m-1) .
\end{aligned}
$$

TheOREM 5.3. If $\alpha+\beta \leqq m+n-2$, and $g_{0}$ is the function of Theorem 5.2, then $g_{0}^{\langle\alpha, \beta\rangle}$ exists and is continuous on $\square$.

This result clearly holds for $g_{0,2}$, hence one need only consider specifically $g_{0,1}$. Let $\alpha+\beta \leqq m+n-2$, and suppose first that $\alpha<n$. If $\beta<m$, then the theorem follows from the fact that $\hat{Y}(x) \check{Z} *(x) \equiv 0$. If $\beta=m-1+j, \quad(j=1, \cdots, n-\alpha-1)$, then use the identity $\hat{Y}(x) \widetilde{Z}^{*}(x) \equiv E_{m}$. On the other hand, if $\beta<m$ and $\alpha=n-1+i$, $(i=1, \cdots, m-\beta-1)$, then use the identity $\tilde{Y}(x) \check{Z} *(x) \equiv-E_{m}$.

THEOREM 5.4. The generalized Green's function for the system (5.2) is not unique. If $u_{1}, \cdots, u_{r}$ form a basis for the solutions of (5.2), $v_{1}, \cdots, v_{\rho}$ form a basis for the solutions of (5.3), and $g_{0}$ is one generalized Green's function for (5.2) then a function $g$ on $\square$ is also a generalized Green's function for (5.2) if and only if there exist essentially bounded and measurable functions $\psi_{1}, \cdots, \psi_{r}, \varphi_{1}, \cdots, \varphi_{p}$ such that if $(x, t) \in \square$, then

$$
g(x, t)=g_{0}(x, t)+\sum_{i=1}^{r} u_{i}(x) \psi_{i}(t)+\sum_{j=1}^{\rho} \varphi_{j}(x) \bar{v}_{j}(t) .
$$

If $g$ is a function on $\square$ satisfying (5.10), then in view of Theorem 
4.3 it follows that $g$ is a generalized Green's function for (5.2).

To establish the converse we may assume without loss of generality that $\left(u_{i}, u_{j}\right)=\delta_{i j},(i, j=1, \cdots, r)$, and $\left(v_{\alpha}, v_{\beta}\right)=\delta_{\alpha \beta},(\alpha, \beta=1, \cdots, \rho)$. If $w \in \mathfrak{A}_{0}$ and $f(x)=w(x)-\sum_{j=1}^{\rho}\left(w, v_{j}\right) v_{j}(x)$, then $\left(f, v_{\alpha}\right)=0,(\alpha=$ $1, \cdots, \rho)$. Thus for this choice of $f$ it follows from Theorem 4.3 that (5.1) has a solution. Suppose that $g$ is a second generalized Green's function for (5.2) and let $d(x, t)=g(x, t)-g_{0}(x, t)$. Then there are constants $\xi_{1}, \cdots, \xi_{r}$ such that

$$
\int_{a}^{b} d(x, t) f(t) d t=\sum_{i=1}^{r} u_{i}(x) \xi_{i}
$$

and if $\Phi(x, t)=d(x, t)-\sum_{j=1}^{p} \bar{v}_{j}(t) \int_{a}^{b} d(x, s) v_{j}(s) d s$, then

$$
\int_{a}^{b} \Phi(x, t) f(t) d t=\sum_{i=1}^{r} u_{i}(x) \xi_{i} \text {. }
$$

Multiplying (5.11) by $\bar{u}_{i}(x)$, and integrating with respect to $x$, we have

$$
\int_{a}^{b} \int_{a}^{b} \bar{u}_{i}(x) \Phi(x, t) f(t) d t d x=\xi_{i}, \quad(i=1, \cdots, r),
$$

and consequently

$$
\int_{a}^{b}\left[\Phi(x, t)-\sum_{i=1}^{r} u_{i}(x) \int_{a}^{b} \bar{u}_{i}(s) \Phi(s, t) d s\right] w(t) d t=0 .
$$

But $w$ is an arbitrary integrable function, and hence

$$
\Phi(x, t)-\sum_{i=1}^{r} u_{i}(x) \int_{a}^{b} \bar{u}_{i}(s) \Phi(s, t) d s=0 \quad \text { on } \square,
$$

and

$$
d(x, t)=\sum_{i=1}^{r} u_{i}(x) \int_{a}^{b} \bar{u}_{i}(s) \Phi(s, t) d s+\sum_{j=1}^{p} \bar{v}_{j}(t) \int_{a}^{b} d(x, s) v_{j}(s) d s .
$$

Hence (5.10) holds with $\psi_{i}$ and $\varphi_{j}$ defined by $\psi_{i}(t)=\int_{a}^{b} u_{i}(s) \Phi(s, t) d s$. and $\varphi_{j}(x)=\int_{a}^{b} d(x, s) v_{j}(s) d s, \quad(i=1, \cdots, r ; j=1, \cdots, \rho)$, and clearly these functions are essentially bounded and measurable.

We now show that a generalized Green's function $g$ for (5.2) has the property that the function $h$ defined by $h(x, t)=\bar{g}(t, x)$ is a generalized Green's function for the adjoint system (5.3). Preliminary to this result we shall prove the following theorem.

Theorem 5.5. Suppose that $u_{1}, \cdots, u_{r}$ form a basis for the solutions of (5.2), $v_{1}, \cdots, v_{\rho}$ from a basis for the solutions of (5.3), and $\Theta=\left\{\theta_{1}, \cdots, \theta_{r}\right\}, \Omega=\left\{\omega_{1}, \cdots, \omega_{p}\right\}$ are sets of integrable functions 
with the property that the matrices $\left[\left(u_{i}, \theta_{j}\right)\right],(i, j=1, \cdots, r)$, and $\left[\left(v_{\alpha}, \omega_{\beta}\right)\right], \quad(\alpha, \beta=1, \cdots, \rho)$, are nonsingular. Then there exists a unique generalized Green's function $g_{L}(, ; \Theta, \Omega)$ for (5.2) satisfying the conditions

$$
\begin{array}{ll}
\int_{a}^{b} g_{L}(x, t ; \Theta, \Omega) \omega_{\alpha}(t) d t=0, & (\alpha=1, \cdots, \rho), \\
\int_{a}^{b} \bar{\theta}_{\imath}(x) g_{L}(x, t ; \Theta, \Omega) d x=0, & (i=1, \cdots, r) .
\end{array}
$$

Without any loss of generality we can assume that $\left[\left(u_{i}, \theta_{j}\right)\right]=E_{r}$ and $\left[\left(v_{\alpha}, \omega_{\beta}\right)\right]=E_{\rho}$. Let $g_{0}$ be the generalized Green's function for (5.2) described in Theorem 5.2. We now determine functions $\psi_{1}, \cdots, \psi_{r}$ and functions $\varphi_{1}, \cdots, \varphi_{\rho}$ such that the generalized Green's function given by (5.10) satisfies conditions (5.12). Such a generalized Green's function $g$ will satisfy the conditions (5.12) if and only if the functions $\gamma_{i},(i=1, \cdots, r)$, and $\varphi_{\alpha},(\alpha=1, \cdots, \rho)$, satisfy the equations

$$
\begin{aligned}
\psi_{i}(x)+\int_{a}^{b} \sum_{\beta=1}^{p} \bar{\theta}_{i}(s) \varphi_{\beta}(s) \bar{v}_{\beta}(x) d s+\int_{a}^{b} \bar{\theta}_{i}(s) g_{0}(s, x) d s & =0, \\
(i & =1, \cdots, r), \\
\varphi_{\alpha}(x)+\int_{a}^{b} \sum_{j=1}^{r} u_{j}(x) \psi_{\jmath}(s) \omega_{\alpha}(s) d s+\int_{a}^{b} g_{0}(x, s) \omega_{\alpha}(s) d s & =0, \\
(\alpha & =1, \cdots, \rho) .
\end{aligned}
$$

A particular set of solutions for equations (5.13) is

$$
\begin{array}{rlrl}
\varphi_{\alpha}(x)= & -\int_{a}^{b} g_{0}(x, s) \omega_{\alpha}(s) d s, & (\alpha=1, \cdots, \rho), \\
\dot{\psi}_{i}(x)= & \int_{a}^{b} \int_{a}^{b} \sum_{\beta=1}^{\rho} \bar{\theta}_{i}(t) g_{0}(t, s) \omega_{\beta}(s) \bar{v}_{\beta}(x) d s d t & \\
& -\int_{a}^{b} \bar{\theta}_{i}(t) g_{0}(t, x) d t, & & (i=1, \cdots, r) .
\end{array}
$$

Moreover, if $\psi_{i}$ and $\varphi_{\alpha},(i=1, \cdots, r ; \alpha=1, \cdots, \rho)$, is any collection of solutions of (5.13), then after substituting the value of $\psi_{i}(x)$ given by the first equation into the second equation of (5.13) it can be shown by straightforward computation that the value of

$$
\sum_{i=1}^{r} u_{i}(x) \psi_{i}(t)+\sum_{\alpha=1}^{p} \varphi_{n}(x) \bar{v}_{\alpha}(t)
$$

is independent of the particular $\psi_{i}$ and $\varphi_{\alpha}$. Hence there is a unique generalized Green's function for (5.2) satisfying (5.12).

The conditions of Theorem 5.5 are clearly satisfied by the sets $\theta_{i}=u_{i},(i=1, \cdots, r)$, and $\omega_{\alpha}=v_{\alpha},(\alpha=1, \cdots, \rho)$; in particular, for linear homogeneous differential operators whose coefficients satisfy 
suitable differentiability conditions, the treatment of Greub and Rheinboldt [4] is limited to this specification.

It is to be remarked that, in view of the definition of $g_{0}$, if $\psi_{i}$ and $\varphi_{\alpha_{2}}(i=1, \cdots, r ; \alpha=1, \cdots, \rho)$, is any collection of solutions of (5.13), then $\varphi_{\alpha} \in \tilde{\mathfrak{A}}_{n},(a=1, \cdots, \rho)$, and $\bar{\psi}_{i} \in \tilde{\mathfrak{P}}_{m},(i=1, \cdots, r)$.

Correspondingly, there exists a unique generalized Green's function $g_{L^{\text {मे }}}(, ; \Omega, \Theta)$ for the system (5.3) which satisfies the conditions

$$
\begin{aligned}
\int_{a}^{b} \bar{\omega}_{\alpha}(x) g_{L^{\grave{\lambda}}}(x, t ; \Omega, \Theta) d x & =0, & & (\alpha=1, \cdots, \rho), \\
\int_{a}^{b} g_{L^{\text {मे }}}(x, t ; \Omega, \Theta) \theta_{i}(t) d t & =0, & & (i=1, \cdots, r) .
\end{aligned}
$$

For brevity, denote by $b_{\Omega}$ and $b_{\theta}$ the functions defined on $\square$ by the formulas

$$
b_{\Omega}(x, t)=\sum_{j=1}^{\rho} \omega_{j}(x) \bar{v}_{j}(t), \quad b_{\theta}(x, t)=\sum_{i=1}^{r} \theta_{i}(x) \bar{u}_{i}(t) .
$$

TheOREM 5.6. If $g_{L}(, ; \Theta, \Omega)$ is the unique generalized Green's function satisfying (5.12), then the following conditions (5.16)-(5.20) are satisfied:

(5.16) $g_{L}^{\langle j, 0\rangle}(, ; \Theta, \Omega),(j=0, \cdots, m+n-2)$, exists and is continuous on $\square$ while $g_{L}^{\langle m+n-1,0\rangle}(x, t ; \Theta, \Omega)$ and $\partial / \partial x g_{L}^{\langle m+n-1,0\rangle}(x, t ; \Theta, \Omega)$ exist on the individual domains $a \leqq t<x, a<x<b$ and $a \leqq x<b, x<t \leqq b$;

(5.17) if $t \in[a, b]$, then the function whose value at $x \neq t$ is $g_{L}^{\langle m+n-1,0\rangle}(x, t ; \Theta, \Omega)$ has a right and a left limit at $t$, denoted by $g_{L}^{\langle m+n-1,0\rangle}\left(t^{+}, t ; \Theta, \Omega\right)$ and $g_{L}^{\langle m+n-1,0\rangle}\left(t^{-}, t ; \Theta, \Omega\right)$, respectively, and

$$
g_{L}^{\langle m+n-1,0\rangle}\left(t^{-}, t ; \Theta, \Omega\right)-g_{L}^{\langle m+n-1,0\rangle}\left(t^{+}, t ; \Theta, \Omega\right)=1 ;
$$

(5.18) if $t \in[a, b]$, then $L\left[g_{L}(, t ; \Theta, \Omega)\right]=b \Omega(, t)$ on $[a, t)$ and $(t, b]$;

(5.19) if $t \in(a, b)$, then the function whose value at $x$ is $g_{L}(x, t ; \Theta, \Omega)$ satisfies the boundary conditions which characterize the set $\mathscr{D}$;

$$
\int_{a}^{b} \bar{\theta}_{i}(x) g_{L}(x, t ; \Theta, \Omega) d x=0, \quad(i=1, \cdots, r ; t \in[a, b]) .
$$

Conditions (5.16)-(5.18) may be verified directly using the properties of $g_{0}$ and the remark following the proof of Theorem 5.5. Condition (5.20) is merely one of the conditions in (5.12). If $\mathscr{D}=\widetilde{\mathfrak{A}}_{n}$, then (5.19) is trivially satisfied. Otherwise, let $w$ be any integrable function, and define $f$ by

$$
f(x)=w(x)-\sum_{\alpha=1}^{p} \omega_{\alpha}(x)\left(w, v_{\alpha}\right)=w(x)-\int_{a}^{b} b_{\Omega}(x, t) w(t) d t .
$$


In view of the assumption that $\left[\left(v_{\alpha}, \omega_{\beta}\right)\right]=E_{\rho}$, it follows that $\left(f, v_{\alpha}\right)=0$, $(\alpha=1, \cdots, \rho)$, and therefore the function $u$ defined by

$$
u(x)=\int_{a}^{b} g_{L}(x, t ; \Theta, \Omega) f(t) d t
$$

is a solution of (5.1). But it follows from (5.12) that

$$
\int_{a}^{b} g_{L}(x, t, \Theta, \Omega) f(t) d t=\int_{a}^{b} g_{L}(x, t ; \Theta, \Omega) w(t) d t
$$

Therefore,

$$
\begin{aligned}
0 & =M \widehat{u}(a)+N \widehat{u}(b) \\
& =\int_{a}^{b}\left(M \widehat{g}_{L}(a, t ; \Theta, \Omega)+N \widehat{g}_{L}(b, t ; \Theta, \Omega)\right) w(t) d t,
\end{aligned}
$$

from which (5.19) follows in view of the arbitrariness of the function $w$.

Corollary. If $w \in \mathfrak{A}_{0}$ and $y$ is defined by

$$
y(x)=\int_{a}^{b} g_{L}(x, t ; \Theta, \Omega) w(t) d t,
$$

then

$$
\begin{aligned}
& L[y]=w-\int_{a}^{b} b_{\Omega}(, t) w(t) d t, \\
& y \in \mathscr{D}, \quad\left(y, \theta_{i}\right)=0, \quad(i=1, \cdots, r) .
\end{aligned}
$$

It should be noted that the unique generalized Green's function $g_{L^{4}}(, ; \Omega, \Theta)$ for (5.3) which satisfies (5.15) also satisfies conditions analogous to $(5.16)-(5.20)$.

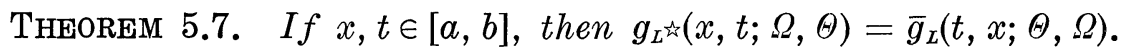

Let $w$ and $h$ be arbitrary integrable functions and define $y$ and $z$ by

$$
\begin{aligned}
& y(x)=\int_{a}^{b} g_{L}(x, t ; \Theta, \Omega) w(t) d t, \\
& z(x)=\int_{a}^{b} g_{L \text { }}(x, t ; \Omega, \Theta) h(t) d t,
\end{aligned}
$$

respectively. Then it follows from the corollary to Theorem 5.6 and its analogue that $y \in \mathscr{D}, z \in \mathscr{D}^{*}$, and therefore

$$
(L[y], z)-\left(y, L^{\text {所 }}[z]\right)=0 .
$$

But it also follows from the corollary to Theorem 5.6 that $L[y]=$ 
$w-\int_{a}^{b} b_{\Omega}(, t) w(t) d t, L^{\sharp}[z]=h-\int_{a}^{b} b_{\theta}(, t) h(t) d t$, and therefore in view of (5.12), (5.15), and the definition of $b_{\Omega}$ and $b_{\theta}$, we have

$$
\int_{a}^{b} \int_{a}^{b} \bar{h}(x)\left[\bar{g}_{L^{\lrcorner}}(t, x ; \Omega, \Theta)-g_{L}(x, t ; \Theta, \Omega] w(t) d t d x=0,\right.
$$

from which the theorem follows since $w$ and $h$ are arbitrary integrable functions.

CoRollary I. The function $g_{L}(, ; \Theta, \Omega)$ is characterized by conditions (5.16)-(5.20), and the function $g_{L^{\not h}}(, ; \Theta, \Omega)$ is characterized by analogous conditions.

As a consequence of Theorems 5.4 and 5.7 one has the following result:

COROLlaRY II. If $g$ is a generalized Green's function for (5.2), then the function $h$ defined by $h(x, t)=\bar{g}(t, x)$ is a generalized Green's function for (5.3).

6. A canonical form for boundary conditions. Let $\left[f_{i j}\right]$ and $\left[g_{i j}\right],(i=0, \cdots, m \geqq 1 ; j=0, \cdots, n \geqq 1)$, be $(m+1) \times(n+1)$ integrable matrix functions. Suppose that the matrix function $\left[f_{i j}\right]$, $(i=0, \cdots, m ; j=0, \cdots, n)$, satisfies hypothesis $(H)$, and $g_{m j}(x) \equiv$ $g_{i n}(x) \equiv 0,(i=0, \cdots, m ; j=0, \cdots, n)$.

For a complex number $\lambda$ let $p_{i j}(; \lambda)$ be the function defined on $[a, b]$ by

$$
p_{i j}(x ; \lambda)=f_{i j}(x)+\lambda g_{i j}(x), \quad(i=0, \cdots, m ; j=0, \cdots, n) .
$$

It follows that for each number $\lambda$ hypothesis $(\mathrm{H})$ holds for the matrix function $\left[p_{i j}(; \lambda)\right]$. For suitable $y$ in $\mathfrak{A}_{n}$ let $\widetilde{y}_{1}(; \lambda), \cdots, \widetilde{y}_{m}(; \lambda)$ be defined on $[a, b]$ as follows:

$$
\begin{aligned}
& \widetilde{y}_{m}(x ; \lambda)=\sum_{j=0}^{n} p_{m j}(x ; \lambda) y^{(j)}(x)=\sum_{j=0}^{n} f_{m j}(x) y^{(j)}(x) ; \\
& \text { if } \widetilde{y}_{i+1}(; \lambda) \in \mathfrak{A}_{1}, \text { then } \widetilde{y}_{i}(x ; \lambda)=\sum_{j=0}^{n} p_{i j}(x ; \lambda) y^{(j)}(x)-\widetilde{y}_{i+1}^{\prime}(x ; \lambda), \\
& (i=m-1, \cdots, 1) .
\end{aligned}
$$

The class of functions $y$ in $\mathfrak{A}_{n}$ for which $\widetilde{y}_{1}(, \lambda), \cdots, \widetilde{y}_{m}(; \lambda)$ are a.c. will be denoted by $\widetilde{\mathfrak{A}}_{n}(\lambda)$, and $L[; \lambda]$ will be the operator with domain $\tilde{\mathfrak{A}}_{n}(\lambda)$, and defined by

$$
L[y ; \lambda]=\sum_{j=0}^{n} p_{0 j}(; \lambda) y^{(j)}-\widetilde{y}_{1}^{\prime}(; \lambda) .
$$


The vector function $\left(\widetilde{y}_{i}(\lambda)\right),(i=1, \cdots, m)$, will be represented by $\widetilde{y}(; \lambda)$, and $\widehat{y}(; \lambda)$ will signify the $(n+m)$-vector function $(y, \cdots$, $\left.y^{(n-1)}, \widetilde{y}_{1}(; \lambda), \cdots, \widetilde{y}_{m}(; \lambda)\right)$. For a complex number $\nu$ let $p_{j i}^{4}(; \nu)$ be the function on $[a, b]$ defined by

$$
p_{j i}^{\not i}(x ; \nu)=\bar{f}_{i j}(x)+\nu \bar{g}_{i j}(x), \quad(i=0, \cdots, m ; j=0, \cdots, n) .
$$

For suitable $z$ in $\mathfrak{A}_{m}$ define $\widetilde{z}_{1}(; \nu), \cdots, \widetilde{z}_{n}(; \nu)$ by

$$
\begin{aligned}
& \widetilde{z}_{n}(x ; \nu)=\sum_{i=0}^{m} p_{n i}^{\dot{i}}(x ; \nu) z^{(i)}(x)=\sum_{i=1}^{m} \bar{f}_{i n}(x) z^{(i)}(x) ; \\
& \text { if } \widetilde{z}_{j+1}(; \nu) \in \mathfrak{N}_{1} \text {, then } \widetilde{z}_{j}(x ; \nu)=\sum_{i=1}^{m} p_{j i}^{\text {市}}(x ; \nu) z^{(i)}(x)-\widetilde{z}_{j+1}^{\prime}(x ; \nu) ; \\
& \quad(j=n-1, \cdots, 1) .
\end{aligned}
$$

The class of functions $z$ in $\mathfrak{A}_{m}$ for which $\widetilde{z}_{1}(; \nu), \cdots, \widetilde{z}_{n}(; \nu)$ are a.c. will be denoted by $\widetilde{\mathfrak{A}}_{m}(\nu)$ and $L^{\sharp}[; \nu]$ will be operator with domain $\tilde{\mathfrak{A}}_{m}(\nu)$, and defined by

$$
L^{\natural}[z ; \nu]=\sum_{i=1}^{m} p_{0 i}^{\varkappa}(; \nu) z^{(i)}-\widetilde{z}_{1}^{\prime}(; \nu) .
$$

The vector function $\left(\widetilde{z}_{j}(; \nu)\right),(j=1, \cdots, n)$, will be represented by $\widetilde{z}(; \nu)$, and $\breve{z}(; \nu)$ will denote the vector function $\left(z, \cdots, z^{(m-1)}, \widetilde{z}_{1}(, \nu), \cdots\right.$, $\left.\widetilde{z}_{n}(; \nu)\right)$. Let $A_{10}, A_{11}, A_{20}$, and $A_{21}$ be $k \times n$ matrices, and let $B_{1}$ and $B_{2}$ be $k \times m$ matrices, $(1 \leqq k \leqq 2 m+2 n-1)$, such that for each number $\lambda$ the $k \times 2(m+n)$ matrix

$$
\left[A_{1}(\lambda)-B_{1} A_{2}(\lambda) B_{2}\right]
$$

has rank $k$, where $A_{1}(\lambda)=A_{10}+\lambda A_{11}$ and $A_{2}(\lambda)=A_{20}+\lambda A_{21}$. Let $\mathscr{D}(\lambda)$ be the collection of functions $y$ in $\tilde{\mathfrak{A}}_{n}(\lambda)$ for which

$$
A_{1}(\lambda) \hat{y}(a)-B_{1} \widetilde{y}(a ; \lambda)+A_{2}(\lambda) \hat{y}(b)+B_{2} \widetilde{y}(b ; \lambda)=0 .
$$

This section is concerned with the particular Euler type quasi-differential system

$$
L[y ; \lambda]=0, \quad y \in \mathscr{D}(\lambda) .
$$

It follows from Theorem 3.2 that the system adjoint to (6.6) is

$$
L^{\sharp}[z ; \bar{\lambda}]=0, \quad z \in \mathscr{D}^{*}(\bar{\lambda}),
$$

where $\mathscr{D}^{*}(\bar{\lambda}) \subset \widetilde{\mathfrak{A}}_{m}(\bar{\lambda})$. The following assumption is made about $\mathscr{D}^{*}(\bar{\lambda})$ :

HYPOTHESIS $\left(\mathrm{H}_{1}\right)$. There exist $(2 m+2 n-k) \times m$ matrices $A_{3}(\nu)=$ $A_{30}+\nu A_{31}, A_{4}(\nu)=A_{40}+\nu A_{41}$ and $(2 m+2 n-k) \times n$ matrices $B_{3}, B_{4}$ such that for arbitrary $\lambda$ the set $\mathscr{D}^{*}(\bar{\lambda})$ is the collection of function $z$ in $\widetilde{\mathfrak{A}}_{m}(\bar{\lambda})$ for which 


$$
A_{3}(\bar{\lambda}) \check{z}(a)-B_{3} \widetilde{z}(a ; \bar{\lambda})+A_{4}(\bar{\lambda}) \check{z}(b)+B_{4} \widetilde{z}(b ; \bar{\lambda})=0 .
$$

It shoud be noted that the assumption used by Zimmerberg to obtain Theorem 2.1 of [13] does not imply that hypothesis $\left(\mathrm{H}_{1}\right)$ holds. For if $m=n=1$ and $k=2 n$, then let the matrices $A_{10}, A_{11}, B_{1}, A_{20}$, $A_{21}, B_{2}$ be defined as

$$
\begin{array}{lll}
A_{10}^{*}=\left[\begin{array}{lll}
1 & 1
\end{array}\right], & \mathrm{A}_{11}^{*}=\left[\begin{array}{lll}
01 & 1
\end{array}\right], & B_{1}^{*}=\left[\begin{array}{ll}
21 \\
]
\end{array}\right], \\
A_{20}^{*}=\left[\begin{array}{ll}
1 & 0
\end{array}\right], & A_{21}^{*}=\left[\begin{array}{ll}
01
\end{array}\right], & B_{2}^{*}=\left[\begin{array}{ll}
01
\end{array}\right] .
\end{array}
$$

Then the hypothesis of Theorem 2.1 of [13] is satisfied, but hypothesis $\left(H_{1}\right)$ does not hold.

If hypothesis $\left(H_{1}\right)$ holds then for each complex number $\nu$ the $(2 m+2 n-k) \times 2(m+n)$ matrix

$$
\left[A_{3}(\nu) B_{3} A_{4}(\nu) B_{4}\right]
$$

has rank $2 m+2 n-k$. Moreover, by a proof quite analogous to that used by Reid to obtain (11.11') of [6] one may establish the following result.

LEMMA 6.1. If hypothesis $\left(H_{1}\right)$ holds, then $\mathscr{D}(\lambda)$ is the collection of functions $y$ in $\widetilde{\mathfrak{A}}_{n}(\lambda)$ for which there is a $(2 m+2 n-k)$-vector $e_{0}$ such that

$$
\begin{array}{ll}
\widehat{y}(a)=B_{3}^{*} e_{0}, & \widetilde{y}(a ; \lambda)=A_{3}^{*}(\bar{\lambda}) e_{0}, \\
\hat{y}(b)=B_{4}^{*} e_{0}, & \widetilde{y}(b ; \lambda)=-A_{4}^{*}(\bar{\lambda}) e_{0},
\end{array}
$$

and $\mathscr{D}^{*}(\bar{\lambda})$ is the collection of functions $z$ in $\tilde{\mathfrak{A}}_{m}(\bar{\lambda})$ for which there is a k-vector $e_{1}$ such that

$$
\begin{array}{ll}
\check{z}(a)=B_{1}^{*} e_{1}, & \widetilde{z}(a ; \bar{\lambda})=A_{1}^{*}(\lambda) e_{1}, \\
\check{z}(b)=B_{2}^{*} e_{1}, & \widetilde{z}(b ; \bar{\lambda})=-A_{2}^{*}(\lambda) e_{1},
\end{array}
$$

where $A_{i}^{*}(\nu)=\left(A_{i}(\nu)\right)^{*},(i=1,2,3,4)$.

Now let $K_{10}=A_{10} B_{3}^{*}+A_{20} B_{4}^{*}, K_{11}=A_{11} B_{3}^{*}+A_{21} B_{4}^{*}, K_{1}(\lambda)=K_{10}+$ $\lambda K_{11}, K_{20}=A_{30} B_{1}^{*}+A_{40} B_{2}^{*}, K_{21}=A_{31} B_{1}^{*}+A_{41} B_{2}^{*}$, and $K_{2}(\lambda)=K_{20}+\lambda K_{21}$. Then the next result follows from Lemma 6.1 and Lemma 3.1.

LemMA 6.2. If hypothesis $\left(H_{1}\right)$ holds, then $K_{2}^{*}(\bar{\lambda})=K_{1}(\lambda)$.

Lemma 6.3. Suppose that hypothesis $\left(H_{1}\right)$ holds, the $k \times 2 m$ matrix $\left[B_{1} B_{2}\right]$ has rank $k-p$, and the $(2 m+2 n-k) \times 2 n$ matrix $\left[B_{3} B_{4}\right]$ has rank $2 m+2 n-k-q$. Then there exist $p \times n$ matrices $\psi_{1}, \psi_{2}$ and $q \times m$ matrices $\psi_{3}, \psi_{4}$ such that the $p \times 2 n$ matrix $\left[\psi_{1} \psi_{2}\right]$ has rank $p$, the $q \times 2 m$ matrix $\left[\psi_{3} \psi_{4}\right]$ has rank $q$, and 


$$
\begin{array}{ll}
\psi_{1} \hat{y}(a)+\psi_{2} \hat{y}(b)=0, & \text { for } y \in \mathscr{D}(\lambda), \\
\psi_{3} \check{z}(a)+\psi_{4} \check{z}(b)=0, & \text { for } z \in \mathscr{D}^{*}(\bar{\lambda}) .
\end{array}
$$

Suppose that $R$ is a $p \times k$ matrix of rank $p$ such that $R\left[B_{1} B_{2}\right]=0$, and define $\psi_{1}$ and $\psi_{2}$ as $\psi_{1}=R A_{10}, \psi_{2}=R A_{20}$. In view of Lemma 6.2 and the fact that for arbitrary complex $\lambda$ the $k \times 2(m+n)$ matrix $\left[A_{1}(\lambda) B_{1} A_{2}(\lambda) B_{2}\right]$ has rank $k$ it follows that there exists a $p \times p$ matrix $V$ such that

$$
\left[R A_{1}(\lambda) R A_{2}(\lambda)\right]=\left(E_{p}+\lambda V\right) R\left[A_{10} A_{20}\right] .
$$

Hence $E_{p}+\lambda V$ is nonsingular and the equation (6.12) is equivalent to

$$
R A_{1}(\lambda) \hat{y}(a)+R A_{2}(\lambda) \hat{y}(b)=0 .
$$

If $R_{0}$ is a $q \times(2 m+2 n-k)$ matrix of rank $q$ such that $R_{0}\left[B_{3} B_{4}\right]=0$, and $\psi_{3}, \psi_{4}$ are defined as $\psi_{3}=R_{0} A_{30}, \psi_{4}=R_{0} A_{40}$, then equation (6.13) may be verified in a similar fashion. The conclusion concerning the ranks of $\left[\psi_{1} \psi_{2}\right]$ and $\left[\psi_{3} \psi_{4}\right]$ is clear.

From Lemma 6.2 it then follows that $\left[B_{1} B_{2}\right]\left[\psi_{3} \psi_{4}\right]^{*}=0$ and $\left[B_{3} B_{4}\right]\left[\psi_{1} \psi_{2}\right]^{*}=0$, so that $q \leqq 2 m-(k-p)$ and $p \leqq 2 n-[2 m+2 n-$ $k-q]=k+q-2 m$, from which one has the following result.

Lemma 6.4. If hypothesis $\left(H_{1}\right)$ holds, then the columns of $\left[\psi_{3} \psi_{4}\right]^{*}$ form $a$ basis for the null space of $\left[B_{1} B_{2}\right]$ and the columns of $\left[\begin{array}{ll}\psi_{1} & \psi_{2}\end{array}\right]^{*}$ form a basis for the null space of $\left[B_{3} B_{4}\right]$.

The following theorem gives a simultaneous canonical representation of the boundary conditions for (6.6) and (6.7) in terms of parameter matrices $\psi_{i}, Q_{i}, G_{i},(i=1,2,3,4)$, and is the central result of this section.

THEOREM 6.1. Suppose that hypothesis $\left(H_{1}\right)$ holds. Then there exist $m \times n$ matrices $Q_{i}$ and $G_{i},(i=1,2,3,4)$, such that $y \in \mathscr{D}(\lambda)$ if and only if there exists a q-vector $\eta_{1}$ such that

$$
\begin{aligned}
\psi_{1} \hat{y}(a)+\psi_{2} \hat{y}(b) & =0, \\
\left(Q_{1}-\lambda G_{1}\right) \hat{y}(a)+\left(Q_{2}-\lambda G_{2}\right) \hat{y}(b)+\psi_{3}^{*} \eta_{1}-\widetilde{y}(a ; \lambda) & =0, \\
\left(Q_{3}-\lambda G_{3}\right) \hat{y}(a)+\left(Q_{4}-\lambda G_{4}\right) \hat{y}(b)+\psi_{4}^{*} \eta_{1}+\widetilde{y}(b ; \lambda) & =0 .
\end{aligned}
$$

Moreover, $z \in \mathscr{D}^{*}(\bar{\lambda})$ if and only if there exists a p-vector $\eta_{2}$ such that

$$
\begin{aligned}
\psi_{3} \check{z}(a)+\psi_{4} \check{z}(b) & =0, \\
\left(Q_{1}^{*}-\bar{\lambda} G_{1}^{*}\right) \check{z}(a)+\left(Q_{3}^{*}-\bar{\lambda} G_{3}^{*}\right) \check{z}(b)+\psi_{1}^{*} \eta_{2}-\widetilde{z}(a ; \bar{\lambda}) & =0, \\
\left(Q_{2}^{*}-\bar{\lambda} G_{2}^{*}\right) \check{z}(a)+\left(Q_{4}^{*}-\bar{\lambda} G_{4}^{*}\right) \check{z}(b)+\psi_{2}^{*} \eta_{2}+\widetilde{z}(b ; \bar{\lambda}) & =0 .
\end{aligned}
$$


Suppose that the matrices $K_{10}$ and $K_{11}$ have ranks $q_{0}$ and $q_{1}$, respectively. Let $D_{10}$ and $D_{11}$ be $(2 m+2 n-k) \times\left(2 m+2 n-k-q_{0}\right)$ and $(2 m+2 n-k) \times\left(2 m+2 n-k-q_{1}\right)$ matrices, respectively, whose individual column vectors form orthonormal bases for the null spaces of $K_{10}$ and $K_{11}$, that is, $K_{10} D_{10}=0$ and $K_{11} D_{11}=0$. As $K_{20}=K_{10}^{*}$ and $K_{21}=K_{11}^{*}$ by Lemma 6.2 , there exist matrices $D_{20}$ and $D_{21}$ of respective orders $k \times\left(k-q_{0}\right)$ and $k \times\left(k-q_{1}\right)$ whose individual column vectors form orthonormal bases for the null spaces of $K_{20}$ and $K_{21}$. Then

$$
\left[\begin{array}{cc}
K_{10} & D_{20} \\
D_{20}^{*} & 0
\end{array}\right], \quad\left[\begin{array}{cc}
K_{11} & D_{21} \\
D_{11}^{*} & 0
\end{array}\right], \quad\left[\begin{array}{cc}
K_{20} & D_{10} \\
D_{20}^{*} & 0
\end{array}\right], \quad\left[\begin{array}{cc}
K_{21} & D_{11} \\
D_{21}^{*} & 0
\end{array}\right]
$$

are nonsingular and have inverses of the form

$$
\left[\begin{array}{cc}
H_{10} & D_{10} \\
D_{20}^{*} & 0
\end{array}\right], \quad\left[\begin{array}{cc}
H_{11} & D_{11} \\
D_{21}^{*} & 0
\end{array}\right], \quad\left[\begin{array}{cc}
H_{10}^{*} & D_{20} \\
D_{10}^{*} & 0
\end{array}\right], \quad\left[\begin{array}{cc}
H_{11}^{*} & D_{21} \\
D_{11}^{*} & 0
\end{array}\right],
$$

respectively. The matrices $H_{10}, H_{11}, H_{10}^{*}$, and $H_{11}^{*}$ are generalized reciprocals of the respective matrices $K_{10}, K_{11}, K_{20}=K_{10}^{*}$, and $K_{21}=K_{11}^{*}$. Let $Q_{i}$ and $G_{i}, \quad(i=1,2,3,4)$, be defined as $Q_{1}=A_{30}^{*} H_{10} A_{10}, Q_{2}=$ $A_{30}^{*} H_{10} A_{20}, Q_{3}=A_{40}^{*} H_{10} A_{10}, Q_{4}=A_{10}^{*} H_{10} A_{20}, G_{1}=-A_{31}^{*} H_{11} A_{11}, G_{2}=-A_{31}^{*} H_{11} A_{21}$, $G_{3}=-A_{41}^{*} H_{11} A_{11}$, and $G_{4}=-A_{41}^{*} H_{11} A_{21}$.

Now if $y \in \mathscr{D}(\lambda)$ then in view of Lemma 6.3 we need only verify the last two equations of (6.14). Suppose that $e_{0}$ is determined by (6.10). Then it follows from (6.10) and the fact that the matrices (6.17) are the inverses of the matrices (6.16) that

$$
\begin{aligned}
& e_{0}=H_{10} A_{10} \hat{y}(a)+H_{10} A_{20} \hat{y}(b)+D_{10} D_{10}^{*} e_{0}, \\
& e_{0}=H_{11} A_{11} \hat{y}(a)+H_{11} A_{21} \hat{y}(b)+D_{11} D_{11}^{*} e_{0} .
\end{aligned}
$$

Now it follows from (6.10) and (6.18) that

$$
\begin{aligned}
\left(Q_{1}-\lambda G_{1}\right) \hat{y}(a) & +\left(Q_{2}-\lambda G_{2}\right) \hat{y}(b)+\left(A_{30}^{*} D_{10} D_{10}^{*}+\lambda A_{31}^{*} D_{11} D_{11}^{*}\right) e_{0} \\
& -\widetilde{y}(a ; \lambda)=0, \\
\left(Q_{3}-\lambda G_{3}\right) \hat{y}(a) & +\left(Q_{4}-\lambda G_{4}\right) \hat{y}(b)+\left(A_{40}^{*} D_{10} D_{10}^{*}+\lambda A_{41}^{*} D_{11} D_{11}^{*}\right) e_{0} \\
& +\widetilde{y}(b ; \lambda)=0 .
\end{aligned}
$$

But $B_{1}\left(A_{30}^{*} D_{10} D_{10}^{*}+\lambda A_{31}^{*} D_{11} D_{11}^{*}\right)+B_{2}\left(A_{40}^{*} D_{10} D_{10}^{*}+\lambda A_{41}^{*} D_{11} D_{11}^{*}\right)=K_{20}^{*} D_{10} D_{10}^{*}+$ $\lambda K_{21}^{*} D_{11} D_{11}^{*}=0$, and consequently the two equations of (6.19) may be written in the form of the last two equations of (6.14) involving the parameter vector $\eta_{1}$.

On the other hand, suppose that $y \in \widetilde{\mathfrak{A}}_{n}(\lambda)$ and (6.14) holds. Now the first equation of (6.14) implies that there is a $(2 m+2 n-k)$-vector $e_{0}$ such that $\hat{y}(a)=B_{3}^{*} e_{0}$ and $\hat{y}(b)=B_{4}^{*} e_{0}$. Hence it follows from (6.16) and (6.17) that (6.18) holds for this value of $e_{0}$. Solving the equations 
(6.18) for $H_{10} A_{10} \hat{y}(a)+H_{10} A_{20} \hat{y}(b)$ and $H_{11} A_{11} \hat{y}(a)+H_{11} A_{21} \hat{y}(b)$, multiplying the first equation on the left by $A_{30}^{*}$ and $A_{40}^{*}$, and the second equation on the left by $\lambda A_{31}^{*}$ and $\lambda A_{41}^{*}$, respectively, and adding it can be shown that the last two equations of (6.14) may be written as

$$
\begin{aligned}
& A_{30}^{*}\left(e_{0}-D_{10} D_{10}^{*} e_{0}\right)+\lambda A_{31}^{*}\left(e_{0}-D_{11} D_{11}^{*} e_{0}\right)+\psi_{3}^{*} \eta_{1}-\widetilde{y}(a ; \lambda)=0, \\
& A_{40}^{*}\left(e_{0}-D_{10} D_{10}^{*} e_{0}\right)+\lambda A_{41}^{*}\left(e_{0}-D_{11} D_{11}^{*} e_{0}\right)+\psi_{4}^{*} \eta_{1}+\widetilde{y}(b ; \lambda)=0 .
\end{aligned}
$$

In view of Lemma 6.2 , the definition of the matrices $D_{10}, D_{11}$, and the choice of the vector $e_{0}$, one sees after multiplying the first equation of $(6.20)$ by $B_{1}$, the second equation by $B_{2}$, and adding the two equations, that $y$ satisfies the boundary conditions of (6.6). The conclusion concerning $D^{*}(\bar{\lambda})$ may be established in a similar manner.

The next theorem is an application of Theorem 6.1, where it is to be noticed that if $m=n$ and $\left[f_{i j}(x)\right],\left[g_{i j}(x)\right]$ are Hermitian, then $\widetilde{\mathfrak{A}}_{n}(\lambda)=\widetilde{\mathfrak{A}}_{n}(\lambda)$; in particular, if $z \in \widetilde{\mathfrak{A}}_{n}(\lambda)$, then $\bar{z}(; \lambda)=\bar{z}(; \lambda)$.

THEOREM 6.2. Suppose that $m=n,\left[f_{i j}(x)\right]$ and $\left[g_{i j}(x)\right]$ are Hermitian on $[a, b], k=2 n$, and $\mathscr{D}^{*}(\bar{\lambda})=\mathscr{D}(\bar{\lambda})$. Then the system (6.6) is equivalent to the Euler-Lagrange equations and transversality conditions for minimizing the functional

$$
\hat{y}^{*}(\alpha)\left[Q_{1} \widehat{y}(a)+Q_{2} \widehat{y}(b)\right]+\hat{y}^{*}(b)\left[Q_{2}^{*} \hat{y}(a)+Q_{4} \widehat{y}(b)\right]+\int_{a}^{b} \sum_{\beta=0}^{n} \bar{y}^{(\alpha)} f_{\alpha \beta} y^{(\beta)},
$$

subject to the restraints

$$
\begin{gathered}
\psi_{1} \hat{y}(a)+\psi_{2} \hat{y}(b)=0, \\
\hat{y}^{*}(a)\left[G_{1} \hat{y}(a)+G_{2} \hat{y}(b)\right]+\hat{y}^{*}(b)\left[G_{2}^{*} \hat{y}(a)+G_{4} \hat{y}(b)\right]+\int_{a}^{b} \sum_{\alpha, \beta=0}^{n-1} \bar{y}^{(\alpha)} g_{\alpha \beta} y^{(\beta)} \\
=\text { const . }
\end{gathered}
$$

If $m=n$, the problem is restricted to the field of real numbers, $g_{i j}(x) \equiv f_{i j}(x) \equiv 0$ for $i \neq j$, and if $f_{i i}, g_{i i} \in \mathfrak{E}_{i},(i, j=0, \cdots, n)$, then the results of this section are the same as obtained by Zimmerberg [12], provided that the formula (2.4) of that paper is corrected by replacing $f_{i}, f_{i+1}, \cdots, f_{n-1}$ by $f_{i}-\lambda g_{i}, f_{i+1}-\lambda g_{i+1}, \cdots, f_{n-1}-\lambda g_{n-1}$, respectively. If, moreover, $g_{i i}(x) \equiv 0$ for $i \geqq 1$, then these are the same results as obtained by Reid [6, Section 11].

7. An application. In this section the results of Section 6 and a theorem of Reid [7] will be used to show that the boundary conditions for' a rather large class of linear $\nu$ th order differential operators may be written in the form given by Theorem 6.1 .

Reid [7] has considered $\nu$ th order linear differential operators $L$ of the form 


$$
L[y]=\sum_{j=0}^{\nu} q_{j}(x) y^{(j)},
$$

with integrable coefficients. Functions $\Lambda_{i}(y ; p),(i=0,1,2, \cdots)$, were defined as

$$
\begin{aligned}
\Lambda_{0}(y ; p) & \equiv p(x) y, \quad \Lambda_{2 r}(y ; p) \equiv\left(p(x) y^{(r)}\right)^{(r)}, \\
\Lambda_{2 r-1}(y ; p) & \equiv \frac{1}{2}\left[\left(p(x) y^{(r-1)}\right)^{(r)}+\left(p(x) y^{(r)}\right)^{(r-1}\right], \quad(r=1,2, \cdots),
\end{aligned}
$$

with the understanding that $p \in \mathfrak{A}_{r}$ in the definition of $\Lambda_{2 r}$ and $\Lambda_{2 r-1}$. The primary result of that paper, and the one of most interest here, is Theorem 3.2 , to the effect that if the polynomials $1, x, \cdots, x^{n} / n$ !, where $n=\nu / 2$ or $n=(\nu+1) / 2$ according as $\nu$ is even or odd, belong to the domain of the adjoint operator $T_{0}^{*}$, then there exist functions $\pi_{j}, \quad(j=0, \cdots, \nu)$, with $\pi_{0} \in \mathfrak{A}_{0}, \pi_{2 \alpha-1} \in \mathfrak{A}_{\alpha}, \pi_{2 \alpha} \in \mathfrak{A}_{\alpha}$ such that $L[y]$ is given by

$$
L[y]=\sum_{j=0}^{\nu} \Lambda_{j}\left(y ; \pi_{j}\right),
$$

while $\mathfrak{A}_{\nu}$ is contained in the domain of the adjoint operator $T_{0}^{*}$ and

$$
T_{0}^{*}(z)=L^{\sharp}[z] \equiv \sum_{j=0}^{\nu} \Lambda_{j}\left(z ;(-1)^{j} \bar{\pi}_{j}\right) \quad \text { for } \quad z \in \mathfrak{A}_{\nu} \text {. }
$$

In view of the differentiability properties of $\pi_{j},(j=1, \cdots, \nu)$, it follows that (7.2) and (7.3) are of the form (6.2) and (6.4), respectively, which in turn reduce to $(2.2)$ and (2.4), respectively, provided that $m=n, g_{i j}(x) \equiv 0$ when $i \geqq 1$ or $j \geqq 1$, and for $i, j=0, \cdots, n$ one defines $f_{i j}(x)$ as follows: $f_{i i}(x)=(-1)^{i} \pi_{2 i}(x) ; f_{i i-1}(x)=(-1)^{i}(1 / 2) \pi_{2 i-1}(x)$, $(i=1, \cdots, n) ; f_{i i+1}(x)=(-1)^{i}(1 / 2) \pi_{2 i+1}(x),(i=0, \cdots, n-1) ; f_{i j}(x) \equiv 0$, $(j<i-1$ and $j>i+1)$.

In particular, if $\nu=2 n$ and $\pi_{2 n}(x) \neq \equiv$, then the vector $\hat{y}(x)$ consists of $y(x)$ and its first $n-1$ derivatives. Similarly, $\check{z}(x)$ consists of $z(x)$ and its first $n-1$ derivatives. The coordinates $\widetilde{y}_{i}(x)$ of the $n$-vector $\widetilde{y}(x)$ are defined by (2.1), and may be expressed in terms of $y(x)$ and its first $2 n-j$ derivatives, $(j=1, \cdots, n-1)$, and similarly for the coordinates of $\widetilde{z}(x)$, defined by (2.3). Consequently, $L[y]$ and

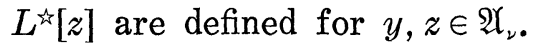

If $\nu=2 n-1$, and $\pi_{\nu}(x) \not \equiv 0$, then $L$ is an operator of odd order and we modify the above defined matrix $\left[f_{i j}(x)\right]$ in the following way: delete the last row, replace $f_{n-1 n}(x)$ with $(-1)^{n-1} \pi_{2 n-1}(x)$, and replace $f_{n-1 n-1}(x)$ with $(-1)^{n-1}\left(\pi_{2 n-2}(x)+(1 / 2) \pi_{2 n-1}^{\prime}(x)\right)$. This change from an $(n+1) \times(n+1)$ matrix $\left[f_{i j}(x)\right]$ to the $n \times(n+1)$ matrix $\left[f_{i j}^{0}\right]$ changes neither the value of $L[y]$ nor the value of $L^{\text {म[ }}[z]$. Now if $\pi_{2 n-1} \in \mathfrak{A}_{n}$, 
then $\pi_{2 n-1}^{\prime} \in \mathfrak{U}_{n-1}$ so that $\tilde{y}_{j}(x)$ may still be differentiated out and written in terms of $y$ and its first $2 n-j$ derivatives, $(j=1, \cdots, n-2)$, and similarly $\widetilde{z}_{i}(x),(i=1, \cdots, n-1)$, may be written in terms of $z(x)$ and its first $2 n-i$ derivatives. Consequently we still have that $L$ and $L^{\text {is }}$ have the common domain $\mathfrak{U}_{\nu}$.

If now it is assumed that there is an $\varepsilon>0$ such that $\left|q_{\nu}(x)\right| \geqq \varepsilon$ almost everywhere, then it follows from Theorem 3.2, or Theorem 4.1 of [7], that the domain of the adjoint operator $T_{0}^{*}$ is $\mathfrak{U}_{\nu}$. Moreover, in view of the formulas which give the canonical variables $\widetilde{y}_{j}(x)$ and $\widetilde{z}_{i}(x)$ in terms of $y(x), \cdots, y^{(n-1)}(x)$ and $z(x), \cdots, z^{(m-1)}(x)$, respectively, we see that there exist nonsingular linear transformations $T$ and $T_{1}$ which transform the vector functions $\left(y, y^{\prime}, \cdots, y^{(\nu-1)}\right)$ and $\left(z, z^{\prime}, \cdots\right.$, $\left.z^{(\nu-1)}\right)$ into the vector functions $\left(y, y^{\prime}, \cdots, y^{(n-1)}, \widetilde{y}_{1}, \cdots, \widetilde{y}_{m}\right)$ and $\left(z, z^{\prime}, \cdots\right.$, $\left.z^{(m-1)}, \widetilde{z}_{1}, \cdots, \widetilde{z}_{n}\right)$, respectively. Therefore, in view of Theorem 3.2 of Reid [7] and Theorem 6.1, it follows that boundary conditions for a $\nu$ th order differential operator of the type described above which involve linearly $y$ and its first $\nu-1$ derivatives at two points may be written as (6.14), and the adjoint boundary conditions may be written as (6.15).

\section{BIBLIOGRAPHY}

1. M. Bôcher, Applications and generalizations of the concept of adjoint systems, Amer. Math. Soc. 14 (1913), 403-420.

2. E. A. Coddington, and N. Levinson, Theory of Ordinary Differential Equations, McGraw-Hill, 1955.

3. W. W. Elliott, Generalized Green's functions for compatible differential systems, Amer. J. Math. 50 (1928), 243-258.

4. W. Greub, and W. C. Rheinboldt, Non-self-adjoint boundary value problems in ordinary differential equations, Journal of Research of the National Bureau of Standards 64B (1960), 83-90.

5. W. T. Reid, Generalized Green's matrices for compatible systems of differential equations, Amer. J. Math. 53 (1931), 443-459.

6. - A new class of self-adjoint boundary value problems, Trans. Amer. Math. Soc. 52 (1942), 381-425.

7. - Adjoint linear differential operators, Trans. Amer. Math. Soc. 85 (1957), $446-461$.

8. - Oscillation criteria for self-adjoint differential systems, Trans. Amer. Math. Soc. 101 (1961), 91-106.

9. - Principal solutions of non-oscillatory linear differential systems, J. Math. Anal. and Appl. 9 (1964), 397-423

10. O. Wyler, Generalized functions of Green for systems of ordinary differential equations, Sandia Corporation Monograph, SCR-98 (1959).

11. On operator solutions of boundary-value problems, Sandia Corporation Monograph, SCR-139 (1959).

12. H. J. Zimmerberg, A self-adjoint differential system of even order, Duke Math. J. 13 (1946), 411-417.

13. _ Two-point boundary problems involving a parameter linearly, Illinois J. Math. 4 (1960), 593-608.

State UNIVERSity of IowA

AND

University of Tennessee 



\section{PACIFIC JOURNAL OF MATHEMATICS}

\section{EDITORS}

\section{H. SAMELSON}

Stanford University

Stanford, California

\section{R. M. BLUMenthaL \\ University of Washington \\ Seattle, Washington 98105}

*J. DugundjI

University of Southern California Los Angeles, California 90007

\section{RICHARD ARENS}

University of California

Los Angeles, California 90024

\section{ASSOCIATE EDITORS}
E. F. BECKENBACH
B. H. NeUManN
F. WOLF
K. YosIDA

\section{SUPPORTING INSTITUTIONS}

\author{
UNIVERSITY OF BRITISH COLUMBIA \\ CALIFORNIA INSTITUTE OF TECHNOLOGY \\ UNIVERSITY OF CALIFORNIA \\ MONTANA STATE UNIVERSITY \\ UNIVERSITY OF NEVADA \\ NEW MEXICO STATE UNIVERSITY \\ OREGON STATE UNIVERSITY \\ UNIVERSITY OF OREGON \\ OSAKA UNIVERSITY \\ UNIVERSITY OF SOUTHERN CALIFORNIA
}

\author{
STANFORD UNIVERSITY \\ UNIVERSITY OF TOKYO \\ UNIVERSITY OF UTAH \\ WASHINGTON STATE UNIVERSITY \\ UNIVERSITY OF WASHINGTON \\ AMERICAN MATHEMATICAL SOCIETY \\ CHEVRON RESEARCH CORPORATION \\ TRW SYSTEMS \\ NAVAL ORDNANCE TEST STATION
}

Mathematical papers intended for publication in the Pacific Journal of Mathematics should be typewritten (double spaced). The first paragraph or two must be capable of being used separately as a synopsis of the entire paper. It should not contain references to the bibliography. Manuscripts may be sent to any one of the four editors. All other communications to the editors should be addressed to the managing editor, Richard Arens at the University of California, Los Angeles, California 90024.

50 reprints per author of each article are furnished free of charge; additional copies may be obtained at cost in multiples of 50 .

The Pacific Journal of Mathematics is published monthly. Effective with Volume 16 the price per volume (3 numbers) is $\$ 8.00$; single issues, $\$ 3.00$. Special price for current issues to individual faculty members of supporting institutions and to individual members of the American Mathematical Society: $\$ 4.00$ per volume; single issues $\$ 1.50$. Back numbers are available.

Subscriptions, orders for back numbers, and changes of address should be sent to Pacific Journal of Mathematics, 103 Highland Boulevard, Berkeley 8, California.

Printed at Kokusai Bunken Insatsusha (International Academic Printing Co., Ltd.), No. 6, 2-chome, Fujimi-cho, Chiyoda-ku, Tokyo, Japan.

PUBLISHED BY PACIFIC JOURNAL OF MATHEMATICS, A NON-PROFIT CORPORATION

The Supporting Institutions listed above contribute to the cost of publication of this Journal, but they are not owners or publishers and have no responsibility for its content or policies.

* Paul A. White, Acting Editor until J. Dugundji returns. 


\section{Pacific Journal of Mathematics}

\section{Vol. 16, No. 2 December, 1966}

Loren N. Argabright, Invariant means on topological semigroups ........ 193

William Arveson, A theorem on the action of abelian unitary groups ...... 205

John Spurgeon Bradley, Adjoint quasi-differential operators of Euler

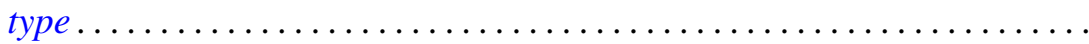

Don Deckard and Lincoln Kearney Durst, Unique factorization in power series rings and semigroups ........................... 239

Allen Devinatz, The deficiency index of ordinary self-adjoint differential operators..................................... 243

Robert E. Edwards, Operators commuting with translations ............ 259

Avner Friedman, Differentiability of solutions of ordinary differential equations in Hilbert space .............................. 267

Boris Garfinkel and Gregory Thomas McAllister, Jr., Singularities in a variational problem with an inequality ......................

Seymour Ginsburg and Edwin Spanier, Semigroups, Presburger formulas,

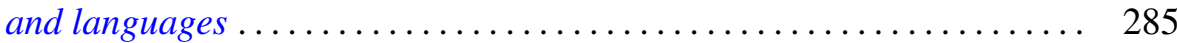

Burrell Washington Helton, Integral equations and product integrals . . . . . . 297

Edgar J. Howard, First and second category Abelian groups with the n-adic topology.........................................

Arthur H. Kruse and Paul William Liebnitz, Jr., An application of a family homotopy extension theorem to ANR spaces.

Albert Marden, I. Richards and Burton Rodin, On the regions bounded by homotopic curves

Willard Miller, Jr., A branching law for the symplectic groups ...

Marc Aristide Rieffel, A characterization of the group algebras of the finite

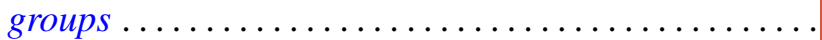

P. P. Saworotnow, On two-sided $H^{*}$-algebras

John Griggs Thompson, Factorizations of p-solvable groups ...

Shih-hsiung Tung, Harnack's inequalities on the classical Cartan

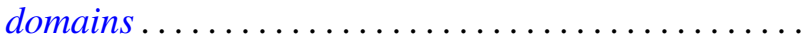

